\title{
Morinda officinalis oligosaccharides alleviate depressive-like behaviors in post-stroke rats via suppressing NLRP3 inflammasome to inhibit hippocampal inflammation
}

\author{
Zhifang Li \\ Wuhan Union Hospital \\ Hexiang Xu \\ Wuhan Union Hospital \\ Yi Xu
}

Beijing tongrentang Co., Ltd. Institute of Science

Guanfeng Lu

Wuhan Union Hospital

Qiwei Peng

Wuhan Union Hospital

Jiefang Chen

Wuhan Union Hospital

Rentang Bi

Wuhan Union Hospital

Jianzhuang Li

Wuhan Union Hospital

Shengcai Chen

Wuhan Union Hospital

Hongkai Li

beijing tongrentang Co., Ltd Institute of Science

Huijuan Jin ( $\sigma_{\text {jinhuijuan1983@163.com ) }}$

Wuhan Union Hospital

Bo Hu

Wuhan Union Hospital

\section{Research}

Keywords: NLRP3 inflammasome, hippocampus, Morinda officinalis oligosaccharides, post-stroke depression 
Posted Date: March 18th, 2021

DOI: https://doi.org/10.21203/rs.3.rs-308551/v1

License: (c) (i) This work is licensed under a Creative Commons Attribution 4.0 International License. Read Full License 


\section{Abstract}

Background: Morinda officinalis oligosaccharides (MOOs) is a traditional Chinese medicine extracted from plant Morinda officinalis roots. It has been used to treat mild and moderate depressive episodes. In the present study, we investigated whether MOOs can ameliorate depressive-like behaviors in post-stroke depression (PSD) rats and further discussed its mechanism by suppressing microglial NLRP3 inflammasome activation to inhibit hippocampal inflammation.

Methods: Behaviors tests were performed to evaluate the effect of MOOs on depressive-like behaviors in PSD rats. The effects of MOOs on the expression of IL-18, IL-1 $\beta$ and nucleotide-binding domain leucinerich repeat family pyrin domain containing 3 (NLRP3) inflammasome were measured in both PSD rats and lipopolysaccharide (LPS)+adenosine triphosphate (ATP) stimulated BV2 cells by reverse transcription polymerase chain reaction (RT-PCR), immunofluorescence and Western blot analysis. Adeno-associated virus (AAV) were injected into hippocampus to downregulate NLRP3 inflammasome expression. The detailed molecular mechanism underlying the effects of MOOs was analyzed by Western blot and immunofluorescence.

Results: MOOs can alleviate depressive-like behaviors in PSD rats. PSD rats showed increased expression of IL-18, IL-1 $\beta$ and NLRP3 inflammasome in the ischemic hippocampus, while MOOs compromised the elevation. NLRP3 downregulation ameliorated depressive-like behaviors and hippocampal inflammation response in PSD rats. Moreover, we found that NLRP3 is mainly expressed on microglia. In vitro, MOOs effectively inhibited the expression of IL-18, IL-1 $\beta$ and NLRP3 inflammasome in LPS+ ATP treated BV2 cells. We further showed that modulation of NLRP3 inflammasome by MOOs was associated with IKB/NF-KB p65 signaling pathway.

Conclusion: Overall, our study revealed the antidepressive effect of MOOs on PSD rats through modulation of microglial NLRP3 inflammasome. We also provide a novel insight into hippocampal inflammation response in PSD pathology and put forward NLRP3 inflammasome as a potential therapeutic target for PSD.

\section{Introduction}

Post-stroke depression (PSD) refers to depression after cerebrovascular incident [1]. The occurrence of PSD is almost one third in stroke survivals [2]. A large number of studies have indicated that PSD is positively associated with poor functional outcome and increased mortality after stroke [3, 4], which gains public concerns. Currently, the treatment of PSD mainly relies on antidepressants such as selective serotonin reuptake inhibitors [5], while side effects remain a problem [6]. Thus, it is urgent to find novel targets for therapeutic approaches of PSD.

In our previous study, we found that PSD rats showed suppressed glucose metabolism due to decreased glucose transporter-3 expression in the neurons of medial prefrontal cortex but not in the hippocampus, which was responsible for the occurrence of PSD [7]. However, hippocampus is demonstrated to be an 
important regulator of emotion and cognition in the brain [8, 9], and evidence has also suggested the correlation among depression, hippocampal inflammation and stroke. Studies have reported high serum level of pro-inflammatory biomarkers in depressive patients $[10,11]$, while several anti-inflammatory agents such as celecoxib have been found to alleviate depressive symptoms in clinical trials [12]. Inhibition of hippocampal inflammation has been proven to ameliorate depressive behaviors in mice [13]. Moreover, cerebral ischemia can lead to widespread inflammatory response in the brain, which can further affect the structure features of the hippocampus [14]. In clinical trials, stroke patients with higher serum IL-18 or IL-1 $\beta$ level at admission have a higher risk of developing PSD $[15,16]$. Thus, excessive hippocampal inflammation may induce depression in stroke survivals.

Nucleotide-binding domain leucine-rich repeat family pyrin domain containing 3 (NLRP3) inflammasome, which is comprised of NLRP3, caspase- 1 and apoptosis-associated speck-like protein (ASC), plays an important role in inflammatory response via regulating the maturation of IL-1 $\beta$ and IL-18 [17]. In the central nervous system, NLRP3 inflammasome mainly expressed on microglia [18] and is reported to be involved in many neurological diseases, including stroke [19], Parkinson's disease [20], and Alzheimer's disease [21]. Selective NLRP3 inflammasome inhibitor has shown to alleviate neurological deficits and reduce IL-1 $\beta$ production in intracerebral hemorrhage mice [22]. Moreover, microglial NLRP3 inflammasome activation in the hippocampus has been found to mediate chronic stress-induced depressive-like behaviors in rats [23]. These findings strongly imply that microglial NLRP3 inflammasome activation is firmly associated with depression. However, whether it is involved in PSD remains unknown.

Morinda officinalis oligosaccharides (MOOs), the traditional Chinese medicine extracted from plant Morinda officinalis roots, has been long used as a tonic to nourish the kidney and strength immunity in human bodies [24]. The Chinese Food and Drug Administration (CFDA) had proven MOOs as a prescribed traditional herbal medicine for mild and moderate depressive episodes in 2012. There have been reports that oral administration of MOOs to depressed rodent animals can increase the monoamine and brainderived neurotrophic factor (BDNF) levels, which suggests the alleviation of depression [24, 25]. In addition, studies have also reported that extracts from Morinda officinalis has effect on colitis by regulating inflammation and cell apoptosis [26, 27].

In the present study, we found that MOOs could ameliorate depressive-like behaviors and hippocampal inflammation in PSD rat models via suppressing microglial NLRP3 inflammasome activation. We further demonstrated the mechanism that MOOs influence the IKB/NF-KB p65 pathway to downregulate NLRP3 inflammasome expression in PSD rats. We also put forward NLRP3 inflammasome as a potential therapeutic target for PSD and provide a novel insight into hippocampal microglial inflammation response in PSD pathology.

\section{Methods}

\section{Establishment of PSD model}


This study was performed under the Institutional Guidelines of the Animal Care and Use Committee, Huazhong University of Science and Technology. To produce the PSD model, transient middle cerebral artery occlusion (tMCAO) operation was performed on Male Sprague-Dawley rats (250-280 g) in accordance with previously described procedures [7]. The filament was withdrawn for reperfusion after 2 hours of tMCAO. Then, rats underwent individual housing and chronic unpredictable mild stress (CUMS) stimulation up to 5 weeks after 7 days' recovery from tMCAO surgery. The CUMS stimuli included water deprivation ( 12 hours), food deprivation ( 24 hours), $45^{\circ}$ cage tilt, tail clamping ( 1 minutes), swimming in $4^{\circ} \mathrm{C}$ water (5 minutes) and overnight illumination ( 36 hours). The sham groups only experienced the same surgical operation, but without tMACO, CUMS stimulation or individual housing.

\section{Behavioral tests}

\section{Tail suspension test (TST)}

Generally, adhesive tape was attached to the tail of rats and the rats were then suspended $50 \mathrm{~cm}$ above the floor and videotaped for 6 minutes. The climbing time and immobility time were recorded as previously described [28].

\section{Forced swimming test (FST)}

On the first day, rats were placed in a transparent cylinder $(50 \mathrm{~cm}$, height; $30 \mathrm{~cm}$, diameter) filled with water ( $30 \mathrm{~cm}$, depth; $25^{\circ} \mathrm{C}$, water temperature) for 6 minutes to adapt to the experiment environment. On the second day, rats were exposed to the same cylinder and videotaped for 6 minutes. The climbing time and immobility time were recorded as previously described [29].

\section{Sucrose preference test (SPT)}

Two bottles containing $1 \%$ sucrose solution were placed into the rats' cages 1 day before the test. On the second day, both bottles were withdrawn and the rats were kept away from water for 4 hours before the test. To start the SPT, one water bottle and one identical $1 \%$ sucrose solution bottle were placed into the rats' cages after weighting both bottles. After 12 hours, the location of the two bottles were switched to avoid location preference. The SPT were lasted for 24 hours and both bottles were reweighed at the end of the test. Sucrose Preference $(\%)=$ Sucrose Volume/(Sucrose Volume + Water Volume) $\times 100 \%$.

\section{Open field test (OFT)}

The OFT was performed to determine the motor ability of rats before other behavioral tests to exclude motor deficiency as previously described [30]. Briefly, rats were placed in a square wooden box $(100 \mathrm{~cm} \times$ $100 \mathrm{~cm} \times 50 \mathrm{~cm}$ ) for 5 minutes. Anilab software (Anilab Scientific Instruments Ltd. Co, Ningbo, China.) was used to analyze traveling distance and average speed of the rats.

\section{Microarray Data}


The microarray data of GSE78731 and GSE124387 were derived from Gene Expression Omnibus (GEO) databases (https://www.ncbi.nlm.nih.gov/geo/query/acc.cgi?acc=GSE78731, and https://www.ncbi.nlm.nih.gov/geo/query/acc.cgi?acc=GSE124387). The dataset GSE78731 included 5 wild-type rats subjected to sham surgery and 5 ischemic rats subjected to MCAO, and its platform was based on the Agilent-028279 SurePrint G3 Rat GE 8x60K Microarray. The dataset GSE124387 included 3 wild-type rats and 3 depressed rats, and its platform was based on the HiSeq X Ten (Rattus norvegicus).

\section{Differential Expression Analysis and Functional Enrichment Analysis}

R software and limma package were used to identify the Differentially Expressed Genes (DEGs) of each GEO dataset. DAVID 6.8 (https://david.ncifcrf.gov/tools.jsp) were applied for functional enrichment analysis of DEGs and top ten biological process terms were selected according to the $P$ value of each term.

\section{Viral injection}

After 2 weeks of tMCAO procedure, rats were aseptically injected with $10 \%$ pentobarbital to induce anesthesia. Then, stereotaxic frames were applied to fix the anesthetized rats. Adeno-associated virus (AAV) solution $\left(1 \times 10^{13} \mathrm{v} . \mathrm{g} . / \mathrm{ml}\right)$ was injected into the hippocampus at the rate of $0.05 \mu \mathrm{L} / \mathrm{min}$ (total volume $2 \mu \mathrm{L}$ ). The coordinates of 4 injection sites: (a) AP, $-3.8 \mathrm{~mm}$; ML, - $1.5 \mathrm{~mm}$; DV, $-3.4 \mathrm{~mm}$; (b) AP, -4.3 mm; ML, -3.0 mm; DV: -3.9 mm; (c) AP, -4.8 mm; ML, -5.2 mm; DV, -3.8 mm; (d) AP, -4.8 mm; ML, -5.2 mm; DV, -6.4 mm. AAV 2/8 serotype (pAKD-CMV-bGlobin-eGFP-H1-shNLRP3, Obio Technology, Shanghai, China) were injected to downregulate NLRP3 expression and null vectors (pAKD-CMV-bGlobin-eGFP-H1shRNA-NC, Obio Technology, Shanghai, China) were used for vehicle control. The behavioral tests were conducted 4 weeks after viral injection.

\section{Cell culture}

The BV2 cell line was purchased from LiScien Biotechnology Ltd. Co., Wuhan, China. BV2 cells were cultured using Dulbecco's modified Eagle's serum (DMEM; HyClone, USA) supplemented with $10 \%$ fetal bovine serum (FBS; Gibco, USA) and $100 \mathrm{U} / \mathrm{mL}$ penicillin-streptomycin (P/S; Thermo Fisher Scientific, Rockford, IL, USA) at $37^{\circ} \mathrm{C}$ in $5 \% \mathrm{CO} 2 / 95 \%$ air. The BV2 cells were subcultured at $1: 2$ ratio and cells culture medium were routinely replaced every 2 days.

\section{Drugs}

MOOs were provided by Beijing Tongrentang Ltd. Co., Beijing, China and dissolved at a concentration of $10 \mathrm{mg} / \mathrm{mL}$ in distilled water. During the last 2 weeks of PSD model establishment, the PSD rats were orally administrated with MOOs at a dose of $0.1 \mathrm{mg} / \mathrm{g} / \mathrm{d}$. The control groups were orally administrated with vehicle of the same volume.

In the cell experiment, MOOs were dissolved at a proper concentration in DMEM. After pre-treatment with MOOs $(0,1.25,2.5$, or $5 \mathrm{mg} / \mathrm{mL}$ ) for 12 hours, BV2 cells were stimulated by LPS (Sigma, Louis, MO, USA) 
for 6 hours and subsequent ATP (Sigma, Louis, MO, USA) for 30 minutes to induce inflammation.

\section{Quantitative real-time PCR}

Total RNA was isolated using TRIzol reagent from Invitrogen. RNA extraction was reverse-transcribed using the cDNA Synthesis Kit (Takara, Kyoto, Japan) according to the manufacturer's protocol, followed by the amplification of the cDNA using the SYBR Premix Ex Taq TM Kit (Takara, Kyoto, Japan). Then, quantitative real-time PCR was performed using the thermocycler (Bio-Rad, Hercules, CA, USA). Primers were shown below.

\begin{tabular}{ll}
\hline Target & Sequence \\
\hline RAT IL-1 $\beta$ Forward & GCACAGTTCCCAACTGGTA \\
RAT IL-1 $\beta$ Reverse & TGTCCGACCATTGCTGTT \\
RAT IL-6 Forward & AGGAGTGGCTAAGGACCAAGACC \\
RAT IL-6 Reverse & TGCCGAGAGACCTCATAGTGACC \\
RAT IL-18 Forward & TGATATCGACCGAACAGCCAACG \\
RAT IL-18 Reverse & GGTCACAGCCAGTCCTCTTACTTC \\
RAT TNF- $\alpha$ Forward & GCATGATCCGAGATGTGGAACTGG \\
RAT TNF- $\alpha$ Reverse & CGCCACGAGCAGGAATGAGAAG \\
RAT NLRP3 Forward & TGATGCATGCACGTCTAATCTC \\
RAT NLRP3 Reverse & CAAATCGAGATGCGGGAGAG \\
RAT ASC Forward & AGAGTCTGGAGCTGTGGCTACTG \\
RAT ASC Reverse & ATGAGTGCTTGCCTGTGTTGTC \\
RAT Caspase1 Forward & GCCCAAGTTTGAAGACAAA \\
RAT Caspase1 Reverse & GGTGTGGAAGAGCAGAAAGC \\
RAT Actin Forward & TGTCACCAACTGGGACGATA \\
RAT Actin Reverse & GGGGTGTTGAAGGTCTCAAA \\
\hline
\end{tabular}

\section{Western Blot}

Total protein was obtained from the microdissected hippocampus and BV2 cells lysis. An equal amount of protein sample was separated by a $12 \%$ SDS-PAGE gel and then transferred onto polyvinylidene fluoride membranes (Merck Millipore, Billerica, MA, USA). The following primary antibodies were used: anti-NLRP3, 1:800, 12446, NOVUS; ASC, 1:300, 1-78977, NOVUS; Caspase1, 1:700, A0964, AbClonal; IL-1ß, 1:700, A16288, AbClonal; IL-18, 1:1000, 10663-1-AP, Proteintech; p65, 1:800, 10745-1-AP, Proteintech; pp65, 1:400, AP0475, AbClonal; IkBa, 1:1000, A1187, AbClonal; p-lkBa, 1:500, AP0707, AbClonal; Actin, 1:1000, AC026, AbClonal; H3, 1:1000, AF0009, Beyotime. Membranes were incubated with primary antibodies overnight at $4^{\circ} \mathrm{C}$ and followed by secondary antibodies. $\beta$-Actin and histone $\mathrm{H} 3$ were used as the housekeeping references for total and nuclear protein. Band intensity was measured with Image $J$ software $(\mathrm{NIH})$. 


\section{Immunofluorescence}

Immunofluorescence was performed as described [7]. The rats' brains were fixed with $4 \%$ paraformaldehyde and then cut into $20 \mathrm{~mm}$ sections. The following primary antibodies were used: antiNLRP3, 1:50, ab4207, Abcam; anti-p65, 1:50, 10745-1-AP, Proteintech; anti-lba-1, 1:200, ab5076, Abcam. Fluorescence-labeled secondary antibodies were applied and 4',6-diamidino-2-phenylindole (DAPI, Invitrogen) was used to stain nuclei. Samples were visualized using a TCS SP5 multiphoton laser scanning confocal microscope (Nikon, Tokyo, Japan).

\section{Statistical analysis}

All values were expressed as mean \pm SEM. Differences between groups were evaluated by the one-way ANOVA of Fisher's least significant difference test or the two-tailed Student's t test using GraphPad Prism 7 software. Values of $P<0.05$ were considered significant, and values of $P<0.01$ were considered markedly significant.

\section{Results}

\section{MOOs alleviate depressive-like behaviours in PSD rats}

To study the anti-depressive effect of MOOs, we used SD rats to establish the PSD model. We randomly divided the SD rats into 4 groups: sham groups administrated with vehicle or MOOs (Ctrl + $v$ group and Ctrl $+\mathrm{M}$ group) and PSD groups administrated with vehicle or MOOs (PSD + v group and PSD + M group). As shown in Fig. $1 A$, we first performed tMACO operation on PSD rats. After 1 week's recovery from surgery, the PSD rats then underwent individual housing and CUMS stimulation up to 5 weeks. We administrated MOOs or vehicle to the PSD rats orally in the last two weeks of PSD model establishment. The sham groups only experienced the same surgical operation, without tMACO, CUMS stimulation or individual housing. Finally, we performed behavioral tests to assess their depressive-like behaviors (Fig. $1 A)$.

From the behavioral tests, we found that depressive-like behaviors were more obvious in PSD rats. The immobility time of PSD $+v$ rats was dramatically increased, while the climbing time was significantly decreased in the TST and FST test compared with Ctrl + v group (Fig. 1B-E). In SPT test, PSD + v rats showed reduced sucrose preference (Fig. $1 F$ ). Orally administration of MOOs to PSD rats can significantly ameliorate the depressive-like behaviors. In the TST and FST test, PSD + M rats showed more climbing time and less immobility time than PSD $+v$ rats (Fig. 1B-E), while the sucrose preference didn't increase in PSD $+M$ rats $($ Fig. $1 F)$, which means that MOOs treatment didn't alleviate anhedonia in PSD rats. To exclude the possibility that motor dysfunction caused by stroke may influence the behavioral tests, we performed OFT test and weighted the rats. No significant difference in traveling distance and average speed was found among the 4 groups in the OFT test and rats' weight in the 4 groups were the same (Fig. 1G-I). From these results, it can be concluded that MOOs can alleviate depressive-like behaviors in PSD rats. 


\section{MO0s inhibit the inflammatory response in the ischemic hippocampus of PSD rats}

Cerebral ischemia can lead to the inflammatory response in the brain, and excessive inflammation, especially in the hippocampus, is found to be associated with depression [13]. Extracts of Morinda officinalis has also been found to regulate intestinal inflammation in mice [26]. Thus, we speculated that MOOs might ameliorate depressive-like behaviors via suppressing inflammatory response in the ischemic hippocampus of PSD rats.

We downloaded the stroke-related gene expression profiling dataset GSE80681 and depression-related gene expression profiling dataset GSE124387 from the GEO database and analyzed the DEGs using R software. Totally, 7956 DEGs were identified in GSE124387 and 3644 DEGs were identified in GSE80681. As showing in Fig. 2A, 819 DEGs were detected in both GSE80681 and GSE124387. Further using DAVID 6.8, we found that the overlapped 819 DEGs were enriched in several inflammation-related biological process (Fig. 2B). The genes involved in the inflammation-related biological process were shown in Table 1. Among them, the levels of IL-1 $1 \beta, I L-18$ as well as TNF- $\alpha$ in serum have been reported to be elevated in PSD patients $[15,16]$. Next, we validated the mRNA expression of IL-1 $\beta$, IL-18 and TNF- $\alpha$ in the rat hippocampus using RT-PCR analysis. As shown in Fig. $2 C-E, P S D+v$ rats showed increased mRNA expression of IL-1 $\beta$, IL-18 and TNF- $\alpha$ in the ischemic hippocampus compared with the control group. MOOs administration significantly reduced IL-1 $\beta$ and IL-18 mRNA expression (Fig. $2 C-D$ ), while TNF-a mRNA expression remained unchanged (Fig. 2E). These results indicated that MOOs may affect IL-1 $\beta$ and IL-18 expression, rather than TNF- $a$ to perform anti-inflammation function. Then, we analyzed the protein expression of IL-1 $\beta$ and IL-18 in the ischemic hippocampus of PSD rats using Western blot analysis. Both IL-1 $\beta$ and IL-18 protein levels were significantly increased in the PSD $+v$ rats compared with the control group, while MOOs administration reversed the change (Fig. $2 F-H)$. From these results, it can be concluded that MOOs treatment can suppress the inflammatory response in the ischemic hippocampus of PSD rats.

\section{MOOs negatively regulate the microglial NLRP3 inflammasome activation in the ischemic hippocampus of PSD rats}

NLRP3 inflammasome, which is comprised of NLRP3, Caspase1 and ASC (Fig. 3A), has been reported to play a crucial role in inflammation by regulating the maturation and release of IL-1 $\beta$ and IL-18 in many diseases [19-21]. In addition, several studies have revealed the association between depression and NLRP3 inflammasome activation [23]. Thus, we proposed that MOOs may alleviate inflammation via suppressing NLRP3 inflammasome activation in the ischemic hippocampus of PSD rats. We measured mRNA and protein expression of NLRP3, Caspase1 and ASC in the hippocampus. PSD $+v$ rats showed significantly increased NLRP3 and Caspase1 mRNA and protein expression, which were reduced in PSD + $\mathrm{M}$ rats (Fig. 3B-H). This result indicated MOOs administration can abolish NLRP3 inflammasome activity in PSD rats. However, no differences in either ASC mRNA or protein expression was obtained among the 4 groups (Fig. 3D and $3 H$ ). We further performed immunofluorescence staining and showed that NLRP3 inflammasome was mainly expressed in Iba- $1^{+}$cells, the normally used marker for microglia (Fig 3/). PSD 
$+v$ rats exhibited increased microglial NLRP3 inflammasome expression in the ischemic hippocampus, while MOOs can reverse the elevation (Fig 3/). These results suggested that MOOs can suppress the microglial NLRP3 inflammasome activation in the hippocampus of PSD rats.

\section{NLRP3 downregulation ameliorates depressive-like behaviors and hippocampal inflammation response in PSD rats}

In order to further demonstrate the participation of NLRP3 inflammasome in the occurrence of PSD, we microinjected PSD rats with AAV-NLRP3 (PSD + AAV-NLRP3) or AAV without NLRP3 sequence (PSD + AAV-null) using stereotaxic frames 2 weeks after tMCAO. Control groups were also injected with AAVNLRP3 (Ctrl + AAV-NLRP3) or AAV without NLRP3 sequence (Ctrl + AAV-null) (Fig 4A-C). After 4 weeks of injection, we found that the NLRP3 expression in the hippocampus of PSD rats was successfully decreased (Fig $5 A-B$ ).

Then, we performed behavioral tests to evaluate the effect of NLRP3 downregulation on depressive-like behaviors of PSD rats. Compared with PSD + AAV-null rats, the immobility time was decreased and the climbing time was increased in PSD + AAV-NLRP3 rats in both TST and FST tests (Fig 4D-G), while Ctrl + AVV-NLRP3 rats showed no changed behavior phenotype (Fig 4D-G). Our data showed that NLRP3 downregulation can ameliorate depressive-like behavior in PSD rats. In accordance to Fig. $1 F$, no difference was detected between PSD + AAV-null and PSD + AAV-NLRP3 on sucrose preference test (Fig 4H). Moreover, the traveling distance and average speed in OFT test and weight showed no difference (Fig $4 /-K$ ), excluding the motor disability among the 4 groups.

To further investigate whether NLRP3 downregulation is adequate to alleviate hippocampal inflammation in PSD rats, we performed Western blot to measure the protein expression of pro-Caspase1, Caspase1 p20, IL-1 $\beta$ and IL-18 in the hippocampus. As shown in Fig 5A-E, AAV-NLRP3 transduction to PSD rats significantly decreased the Caspase $1 \mathrm{p} 20, \mathrm{IL}-1 \beta$ and IL-18 expression in the hippocampus compared with PSD + AAV-null rats without affecting expression of pro-Caspase1. Transduction of AAV-NLRP3 in control rats did not influence the Caspase $1 \mathrm{p} 20, \mathrm{IL}-1 \beta$ and IL-18 protein expression (Fig $5 C$ - $E$ ). Taken together, these results confirmed that downregulating NLRP3 was adequate to abrogate hippocampal inflammation response in PSD rats.

\section{MOOs inhibit IKB/NF-KB/NLRP3 signaling pathway in LPS+ATP treated BV2 cells}

Then, we tested the effect of MOOs on NLRP3 inflammasome activation in BV2 cells in vitro. BV2 cells were treated with LPS for 6 hours followed by ATP for 0.5 hour to induce inflammatory response. Meanwhile, MOOs were incubated at concentrations of $0,1.25,2.5$, and $5 \mathrm{mg} / \mathrm{mL}$ in culture medium. As shown in Fig 6A-G, LPS+ATP stimuli significantly increased the expression of NLRP3, Caspase1 p20, IL$1 \beta$ and IL-18 in BV2 cell lysate and caspase1 p20 and IL-1 $\beta$ in the supernatant, which suggested LPS+ATP treatment induced NLRP3 inflammasome activation. MOOs administration significantly reduced NLRP3, Caspase1 p20, IL-1 $\beta$ and IL-18 expression in cell lysate, as well as Caspase1 p20 and IL$1 \beta$ concentration in supernatant in a dose-dependent manner, without affecting the expression of pro- 
Caspase1 (Fig 6A-G). These results indicated that MOOs can suppress LPS+ATP induced NLRP3 inflammasome activation in BV2 cells.

Previous studies have demonstrated that NLRP3 inflammasome activation can be regulated by transcriptional factor NF-kB [17]. Thus, we measured the plкB, IкB, pNF-kB p65 and NF-kB p65 protein expression in LPS+ATP treated BV2 cells in the presence or absence of MOOs. We found that levels of

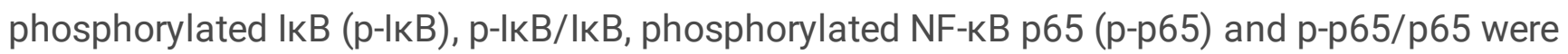
significantly increased in LPS+ATP treated BV2 cells, which indicated the activation of $1 \mathrm{~KB} / \mathrm{NF}-\mathrm{kB}$ signaling pathway. MOOs treatment can reverse these changes, while total IKB and NF-KB p65 levels were not influenced (Fig 7A-G). The activated NF-KB p65 can translocated into the nucleus initiating the transcription. Accordingly, we found that nucleus p65 (np65) level was increased after LPS+ATP treatment, while MOOs significantly abrogated the effect (Fig 7A, $H$ ). Confocal microscopy also showed that MOOs treatment can lessen the nuclear translocation of NF-KB p65 in LPS+ATP treated BV2 cell (Fig 7I). In conclusion, MOOs may inhibit the IKB/NF-KB signaling pathway to negatively regulate NLRP3 inflammasome in BV2 cells.

\section{MOOs suppress IKB/NF-KB signaling pathway in PSD rats}

To further confirm the effect of MOOs on IKB/NF-KB signaling pathway in vivo, we measured the protein expression of $p-I_{k} B, I K B, p-p 65, p 65$ and np65 in the ischemic hippocampus of PSD rats. We found that compared to the control group, the levels of $p-I_{\kappa} B, p-I_{k} B / \mathrm{k} B, p-p 65, p-p 65 / p 65$ and np65 were increased in PSD $+v$ rats. MOOs can reverse the elevation without affecting total IKB and p65 level (Fig $8 A$ $H$ ). Further confocal microscopy was also preformed to assess the nuclear translocation of p65 in microglia in PSD rats. We found that PSD $+v$ rats showed higher nuclear translocation of $p 65$ in microglia than $\mathrm{Ctrl}+\mathrm{v}$ rats and MOOs can reduce this translocation (Fig 8). Taken together, MOOs may inhibit NLRP3 inflammasome activation of PSD rats by suppressing the IKB/NF-KB signaling pathway.

\section{Discussion}

In this study, we demonstrated the antidepressive effect of MOOs, the herbal extracts from plant Morinda officinalis roots, in PSD rat models. We also revealed the mechanism that MOOs alleviate hippocampal inflammation response by inhibiting microglial NLRP3 inflammasome activation. We further identified that MOOs may suppress the IKB/NF-kB p65 pathway to downregulate NLRP3 inflammasome expression. The results indicated that NLRP3 inflammasome is a potential target for PSD treatment, and highlighted the involvement of NLRP3 inflammasome activation in PSD pathology.

Morinda officinalis is one of the best-known Chinese herbs and widely used as a Yang-tonic agent to treat a wide range of diseases, such as osteoporosis, rheumatoid arthritis, impotence, dermatitis, Alzheimer's disease and depression [24]. Phytochemistry studies revealed that morinda officinalis is comprised of variable chemical constituents, mainly including iridoid glycosides, anthraquinones, polysaccharides, oligosaccharides, organic acids, and volatile oils [24]. MOOs are the inulin-type fructooligosaccharides extracted from morinda officinalis, with 3-9 degrees of polymerization (DPs). Among them, the DP5 has 
the largest proportion of MOOs [31]. Previous studies have reported that oral administration of MOOs can increase the monoamine and BDNF levels in rodent depression model, which suggests its effect on depression [24, 25]. In 2012, CFDA had approved MOOs as a prescribed medicine for minor or moderate depression. In our studies, we further demonstrated the effect of MOOs on alleviating depressive-like behaviors in PSD rats, which may provide evidence for its clinical treatment of PSD patients. Of note, the blood brain barrier is injuried with high permeability after cerebral ischemic insult, even up to 1 month after stroke in rats [32], thus the orally administrated MOOs can finally reach the brain to perform function.

Hippocampal inflammation has been extensively investigated in depression. Inhibition of hippocampal inflammation can alleviate LPS-induced depressive behaviors in mice [13]. Moreover, several clinical trials have illustrated that stroke patients with higher serum IL-1 $\beta$ or IL-18 level at admission have a higher risk of developing PSD $[15,16]$. Here, we further demonstrated that inflammatory response was activated in the hippocampus of PSD rats. The microglial NLRP3 inflammasome activation was responsible for the excessive inflammatory response. In consistent with previous studies [18], our study confirmed that NLRP3 inflammasome is mainly activated in microglia in the hippocampus of PSD rats. Downregulation of hippocampal NLRP3 inflammasome abrogated depressive-like behaviors and mitigated inflammatory response in the ischemic hippocampus of PSD rats. Although our findings cannot fully elucidate the mechanism of PSD, they offer another insight into the correlation among hippocampal inflammatory response, microglial NLRP3 inflammasome activation and the occurrence of PSD.

We further explored how MOOs regulate NLRP3 inflammasome and determined NF-KB signaling pathway. When suffer with extracellular stimuli, ІкB can be phosphorylated by kinase and subsequently dislocated from the NF-KB dimer, followed by the translocation of NF-KB into cell nucleus [33]. Nuclear NF-KB can promote the transcription of NLRP3 initiating NLRP3 inflammasome activation [34]. Additionally, NF-KB signaling pathway was activated in both depression [35] and ischemic situations [36]. In this study, MOOs suppressed the increase of phosphorylated ІкB and NF-KB p65 in both PSD rats and BV2 cells, which could be involved in inhibited NLRP3 inflammasome activation. Notably, the regulation of NLRP3 inflammasome activation is a complex process and many other factors may also be involved, such as trans-Golgi disassembly, lysosomal disruption and $\mathrm{K}^{+}$efflux [17]. Thus, further investigation is needed to fully clarify the mechanism of MOOs on NLRP3 inflammasome activation inhibition.

One of the limitations of our studies is that the AAV-NLRP3 we designed to downregulate hippocampal NLRP3 inflammasome in our work was not specifically targeted to microglia. Other cell types such as astrocytes and neurons may also be transfected with AAV-NLRP3, resulting in decreased reliability of the results. However, in consistent with other studies [18, 37], our work revealed that NLRP3 is mainly expressed on microglia. Thus, it is possible that AAV-NLRP3 mainly downregulate NLRP3 in microglia. Future studies should be designed to specifically downregulate microglial NLRP3 inflammasome to verify our results. Additionally, we didn't identify the specific molecular target of MOOs, because the signal component of MOOs cannot be purified currently due to limitations in purification technology. 
In conclusion, this study revealed the antidepressive effect and pharmacological mechanism of MOOs, and emphasized NLRP3 inflammasome as the possible therapeutic target of PSD. We further illustrated that MOOs alleviate depressive-like behaviors in PSD rats through the IKB/NF-KB p65/NLRP3 inflammasome signaling pathway, which is an inflammatory pathway and may also offer insights for PSD treatment. Moreover, we put forward the correlation among stroke, hippocampal inflammation, and PSD. We further proposed the viewpoint that hippocampal inflammatory response activation followed by stroke could play a crucial role in the pathogenesis of PSD, and that alleviating hippocampal inflammation through downregulating NLRP3 inflammasome is a potential novel target for PSD.

\section{Conclusion}

In this study, we confirmed the antidepressive effect of MOOs in PSD rat models. We also identified that MOOs can suppress microglial NLRP3 inflammasome activation to alleviate hippocampal inflammation. Moreover, we further illustrated that the IKB/NF-KB p65 pathway can be inhibited by MOOs to downregulate NLRP3 inflammasome expression. The results shed light on NLRP3 inflammasome as a potential target for PSD treatment, and highlighted the involvement of hippocampal inflammation in PSD pathology.

\section{Abbreviations}

MOOs Morinda officinalis oligosaccharides

PSD poststroke depression

NLRP3 nucleotide-binding domain leucine-rich repeat family pyrin domain containing 3

LPS lipopolysaccharide

ATP adenosine triphosphate

RT-PCR reverse transcription polymerase chain reaction

AAV adeno-associated virus

ASC apoptosis-associated speck-like protein

CFDA Chinese Food and Drug Administration

BDNF brain-derived neurotrophic factor

tMCAO transient middle cerebral artery occlusion

CUMS chronic unpredictable mild stress 
TST tail suspension test

FST forced swim test

SPT sucrose preference test

OFT open field test

GEO Gene Expression Omnibus

DEGs Differentially Expressed Genes

DP degrees of polymerization

\section{Declarations}

\section{Ethics approval and consent to participate}

All rats experiments were approved by the Medical Ethics Committee of Tongji Medical College and all experimental procedures were conducted in accordance with the Institutional Guidelines of the Animal Care and Use Committee, Huazhong University of Science and Technology.

\section{Consent for publication}

Not applicable.

\section{Availability of data and materials}

The datasets used in the current study are applicable from the corresponding author on reasonable request.

\section{Competing interests}

The authors declare that they have no conflict of interests.

\section{Funding}

This work was supported by the National Natural Science Foundation of China (Grants: 81571119 to BH, 81671147 to $\mathrm{JHJ}$ ), Major refractory diseases pilot project of clinical collaboration with Chinese \& Western Medicine (SATCM-20180339) and National Key Research and Development Program of China (2018YFC1312200 to Bo Hu).

\section{Authors' contributions}

ZL and HX performed major experiments; GL performed the bioinformatic analysis; QP, JC analyzed data; RB and JL performed behavior tests; SC, YX, and HL reviewed the article; HJ and BH conceived the study 
and reviewed the article.

\section{Acknowledgements}

The authors thank the Neurology and Biochemistry Laboratory of Union Hospital, Tongji Medical College, Huazhong University of Science and Technology, for providing the experimental facilities.

\section{References}

1. Dwyer Hollender, K., Screening, diagnosis, and treatment of post-stroke depression. J Neurosci Nurs, 2014. 46(3): p. 135-41.

2. Hackett, M.L. and K. Pickles, Part l: frequency of depression after stroke: an updated systematic review and meta-analysis of observational studies. Int J Stroke, 2014. 9(8): p. 1017-25.

3. Bartoli, F., et al., Depression after stroke and risk of mortality: a systematic review and meta-analysis. Stroke Res Treat, 2013. 2013: p. 862978.

4. Blochl, M., S. Meissner, and S. Nestler, Does depression after stroke negatively influence physical disability? A systematic review and meta-analysis of longitudinal studies. J Affect Disord, 2019. 247: p. 45-56.

5. Villa, R.F., F. Ferrari, and A. Moretti, Post-stroke depression: Mechanisms and pharmacological treatment. Pharmacol Ther, 2018. 184: p. 131-144.

6. Collaboration, F.T., Effects of fluoxetine on functional outcomes after acute stroke (FOCUS): a pragmatic, double-blind, randomised, controlled trial. Lancet, 2019. 393(10168): p. 265-274.

7. Zhu, J., et al., Morinda officinalis oligosaccharides ameliorate depressive-like behaviors in poststroke rats through upregulating GLUT3 to improve synaptic activity. FASEB J, 2020. 34(10): p. 1337613395.

8. Poppenk, J., et al., Long-axis specialization of the human hippocampus. Trends Cogn Sci, 2013. 17(5): p. 230-40.

9. Fanselow, M.S. and H.W. Dong, Are the dorsal and ventral hippocampus functionally distinct structures? Neuron, 2010. 65(1): p. 7-19.

10. Dahl, J., et al., The plasma levels of various cytokines are increased during ongoing depression and are reduced to normal levels after recovery. Psychoneuroendocrinology, 2014. 45: p. 77-86.

11. Wium-Andersen, M.K., et al., Elevated C-reactive protein levels, psychological distress, and depression in 73, 131 individuals. JAMA Psychiatry, 2013. 70(2): p. 176-84.

12. Nery, F.G., et al., Celecoxib as an adjunct in the treatment of depressive or mixed episodes of bipolar disorder: a double-blind, randomized, placebo-controlled study. Hum Psychopharmacol, 2008. 23(2): p. 87-94.

13. Li, M., et al., Lentivirus-mediated interleukin-1beta (IL-1beta) knock-down in the hippocampus alleviates lipopolysaccharide (LPS)-induced memory deficits and anxiety-and depression-like behaviors in mice. J Neuroinflammation, 2017. 14(1): p. 190. 
14. Nikonenko, A.G., et al., Structural features of ischemic damage in the hippocampus. Anat Rec (Hoboken), 2009. 292(12): p. 1914-21.

15. Kim, J.M., et al., Associations of Tumor Necrosis Factor-alpha and Interleukin-1beta Levels and Polymorphisms with Post-Stroke Depression. Am J Geriatr Psychiatry, 2017. 25(12): p. 1300-1308.

16. Kang, H.J., et al., Effects of interleukin-6, interleukin-18, and statin use, evaluated at acute stroke, on post-stroke depression during 1-year follow-up. Psychoneuroendocrinology, 2016. 72: p. 156-60.

17. Swanson, K.V., M. Deng, and J.P. Ting, The NLRP3 inflammasome: molecular activation and regulation to therapeutics. Nat Rev Immunol, 2019. 19(8): p. 477-489.

18. Gustin, A., et al., NLRP3 Inflammasome Is Expressed and Functional in Mouse Brain Microglia but Not in Astrocytes. PLoS One, 2015. 10(6): p. e0130624.

19. Ma, D.C., et al., Kv1.3 channel blockade alleviates cerebral ischemia/reperfusion injury by reshaping M1/M2 phenotypes and compromising the activation of NLRP3 inflammasome in microglia. Exp Neurol, 2020. 332: p. 113399.

20. Haque, M.E., et al., Targeting the microglial NLRP3 inflammasome and its role in Parkinson's disease. Mov Disord, 2020. 35(1): p. 20-33.

21. Heneka, M.T., et al., NLRP3 is activated in Alzheimer's disease and contributes to pathology in APP/PS1 mice. Nature, 2013. 493(7434): p. 674-8.

22. Ren, H., et al., Selective NLRP3 (Pyrin Domain-Containing Protein 3) Inflammasome Inhibitor Reduces Brain Injury After Intracerebral Hemorrhage. Stroke, 2018. 49(1): p. 184-192.

23. Feng, X., et al., Glucocorticoid-Driven NLRP3 Inflammasome Activation in Hippocampal Microglia Mediates Chronic Stress-Induced Depressive-Like Behaviors. Front Mol Neurosci, 2019. 12: p. 210.

24. Zhang, J.H., et al., Morinda officinalis How. - A comprehensive review of traditional uses, phytochemistry and pharmacology. J Ethnopharmacol, 2018. 213: p. 230-255.

25. Xu, L.Z., et al., BDNF-GSK-3beta-beta-Catenin Pathway in the mPFC Is Involved in Antidepressant-Like Effects of Morinda officinalis Oligosaccharides in Rats. Int J Neuropsychopharmacol, 2017. 20(1): p. 83-93.

26. Liang, J., et al., The Extracts of Morinda officinalis and Its Hairy Roots Attenuate Dextran Sodium Sulfate-Induced Chronic Ulcerative Colitis in Mice by Regulating Inflammation and Lymphocyte Apoptosis. Front Immunol, 2017. 8: p. 905.

27. Shin, J.S., et al., Monotropein isolated from the roots of Morinda officinalis ameliorates proinflammatory mediators in RAW 264.7 macrophages and dextran sulfate sodium (DSS)-induced colitis via NF-kappaB inactivation. Food Chem Toxicol, 2013. 53: p. 263-71.

28. Yan, Y., et al., Effect of oxytocin on the behavioral activity in the behavioral despair depression rat model. Neuropeptides, 2014. 48(2): p. 83-9.

29. Detke, M.J. and I. Lucki, Detection of serotonergic and noradrenergic antidepressants in the rat forced swimming test: the effects of water depth. Behav Brain Res, 1996. 73(1-2): p. 43-6. 
30. Goncalves, F.M., et al., Glutamatergic system and mTOR-signaling pathway participate in the antidepressant-like effect of inosine in the tail suspension test. J Neural Transm (Vienna), 2017. 124(10): p. 1227-1237.

31. Cha, X., et al., Inulin with a low degree of polymerization protects human umbilical vein endothelial cells from hypoxia/reoxygenation-induced injury. Carbohydr Polym, 2019. 216: p. 97-106.

32. Garbuzova-Davis, S., et al., Compromised blood-brain barrier competence in remote brain areas in ischemic stroke rats at the chronic stage. J Comp Neurol, 2014. 522(13): p. 3120-37.

33. Hayden, M.S. and S. Ghosh, NF-kappaB in immunobiology. Cell Res, 2011. 21(2): p. 223-44.

34. Bauernfeind, F.G., et al., Cutting edge: NF-kappaB activating pattern recognition and cytokine receptors license NLRP3 inflammasome activation by regulating NLRP3 expression. J Immunol, 2009. 183(2): p. 787-91.

35. Xu, Y., et al., NLRP3 inflammasome activation mediates estrogen deficiency-induced depression- and anxiety-like behavior and hippocampal inflammation in mice. Brain Behav Immun, 2016. 56: p. 17586.

36. Yang, C.H., et al., Multi-Targeting Andrographolide, a Novel NF-kappaB Inhibitor, as a Potential Therapeutic Agent for Stroke. Int J Mol Sci, 2017. 18(8).

37. Houtman, J., et al., Beclin1-driven autophagy modulates the inflammatory response of microglia via NLRP3. EMBO J, 2019. 38(4).

\section{Table}

Table 1: Inflammation-related terms in the top ten biological process terms according to the $\mathrm{P}$ value of each term 


\begin{tabular}{|c|c|c|}
\hline Terms & Counts & Genes \\
\hline $\begin{array}{l}\text { cellular response } \\
\text { to tumor necrosis } \\
\text { factor }\end{array}$ & 25 & $\begin{array}{l}\text { CD40, CALCA, CCL11, ADAMTS12, ICAM1, PYCARD, CCL9, CCL7, } \\
\text { CCL6, CYP11A1, CCL4, CCL3, CCL2, CYP1B1, HAS2, CCL19, } \\
\text { ADAMTS7, ENTPD1, ZFP34, IL18, CYBA, MMP9, COL1A1, FABP4, } \\
\text { LCN2 }\end{array}$ \\
\hline $\begin{array}{l}\text { chemokine- } \\
\text { mediated } \\
\text { signaling pathway }\end{array}$ & 17 & $\begin{array}{l}\text { CCL11, GPR35, CXCR6, CXCL13, CXCL3, CXCL10, CCL9, CXCL11, } \\
\text { CCL7, CCL6, CXCR3, CCL4, CCL3, CCL2, CCR6, CCL19, PF4 }\end{array}$ \\
\hline $\begin{array}{l}\text { cellular response } \\
\text { to } \\
\text { lipopolysaccharide }\end{array}$ & 27 & $\begin{array}{l}\text { CD86, CSF3, CD40, CEBPE, LY96, CXCL3, TNF, CXCL16, ICAM1, } \\
\text { PYCARD, KLRK1, THPO, PLAU, CYP11A1, MRC1, CCL2, STAP1, CD36, } \\
\text { LBP, GBP2, ENTPD1, IL18, MMP9, CXCL10, IL1 } \boldsymbol{\beta} \text {, LCN2 }\end{array}$ \\
\hline $\begin{array}{l}\text { response to } \\
\text { lipopolysaccharide }\end{array}$ & 35 & $\begin{array}{l}\text { CD86, IL1RN, CD40, PTGER1, LY96, MGST2, ADM, CXCL13, CXCL3, } \\
\text { TNF, LOXL1, C2, ICAM1, ADH4, THBD, EDNRA, THPO, CASP1, CCL2, } \\
\text { LBP, JUNB, ENTPD1, SERPINA3N, IGF1, MMP9, CXCL10, CXCL11, } \\
\text { SLPI. TH IL1B. CFB, PF4, PTGES. FGF10 }\end{array}$ \\
\hline $\begin{array}{l}\text { cellular response } \\
\text { to interleukin-1 }\end{array}$ & 19 & $\begin{array}{l}\text { CD40, CCL11, PTGIS, SERPINA3N, ADAMTS12, MMP9, ICAM1, } \\
\text { PYCARD, CCL9, CCL7, CCL6, CYP11A1, CCL4, LCN2, CCL3, CCL2, } \\
\text { HAS2, CCL19, ADAMTŚ7 }\end{array}$ \\
\hline $\begin{array}{l}\text { positive } \\
\text { regulation of } \\
\text { inflammatory } \\
\text { response }\end{array}$ & 15 & $\begin{array}{l}\text { STAT5A, CCL11, ITGA2, TNF, IL17RB, CCL9, EDNRA, FABP4, CCL7, } \\
\text { CCL6, CCL4, CCL3, CCL2, TLR10, TGM2 }\end{array}$ \\
\hline $\begin{array}{l}\text { inflammatory } \\
\text { response }\end{array}$ & 33 & $\begin{array}{l}\text { UCN, CD40, CALCA, PTGER1, CXCR6, CXCL13, CXCL3, TNF, KNG1, } \\
\text { PYCARD, CCL9, CCL7, CCL6, CXCR3, CCL4, CCL3, SPP1, CCL2, } \\
\text { CCL19, ITGB6, ANXA1, IL18, APOC3, CELA1, CYBA, SERPINA3N, } \\
\text { CXCL10, CXCL11, IL13, CRH, TLR10, TLR6, PF4 }\end{array}$ \\
\hline
\end{tabular}

\section{Figures}


A

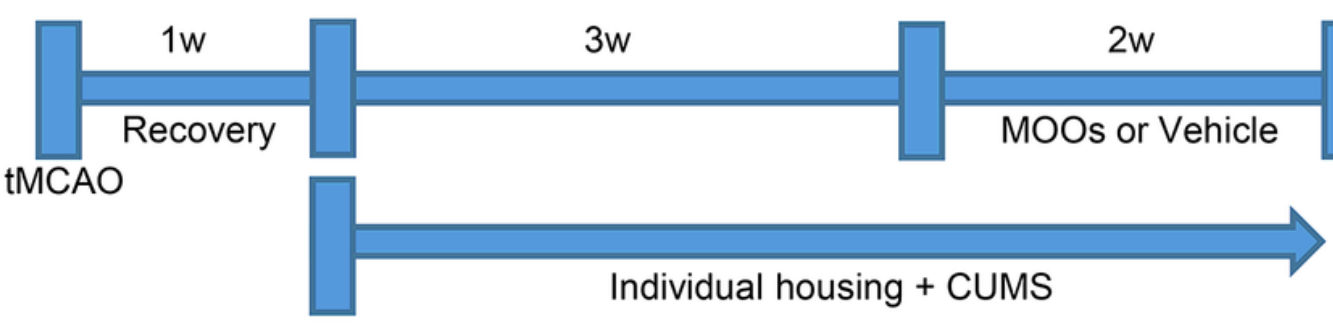

B

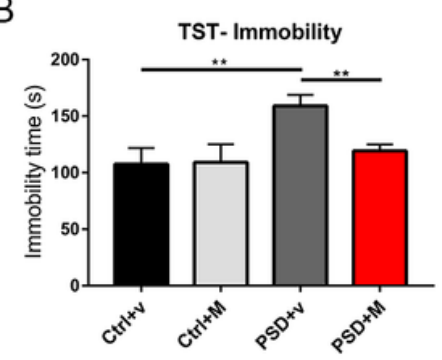

F

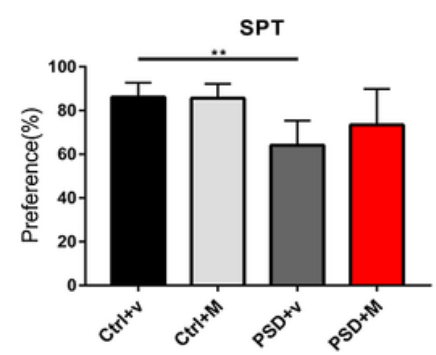

C

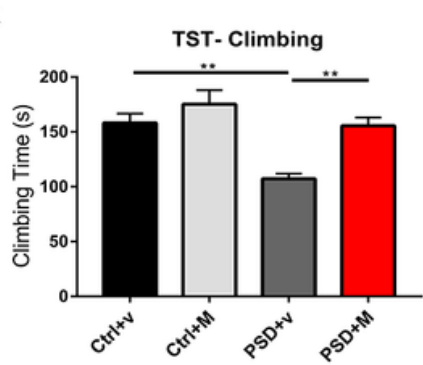

G

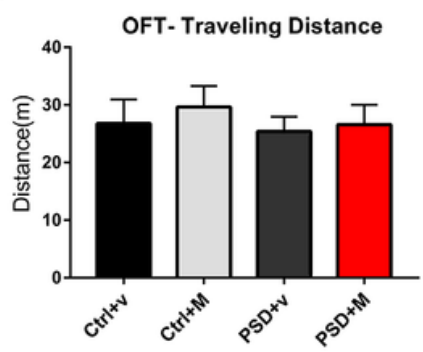

D

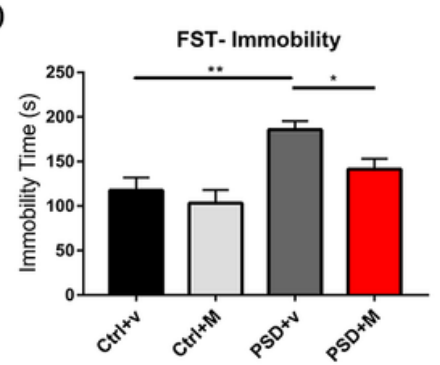

$\mathrm{H}$

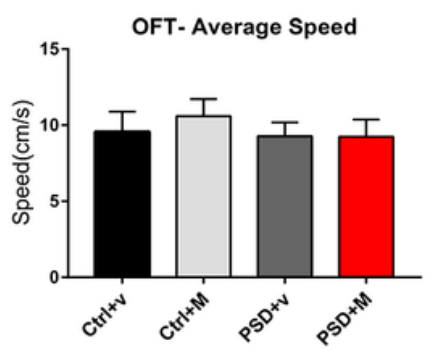

Behavioural tests

E

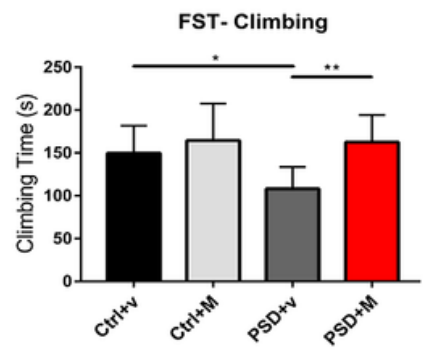

I

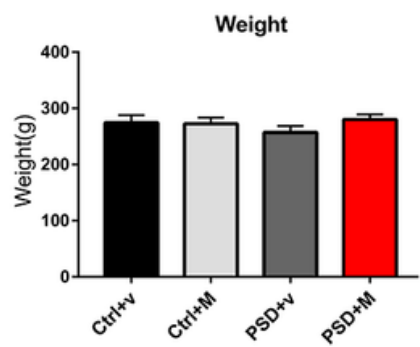

Figure 1

Morinda officinalis oligosaccharides (MOOs) alleviate depressive-like behaviors in PSD rats. A An illustration of the experimental schedule. $B, C$ The effect of MOOs on the immobility time (B) and the climbing time (C) in the TST test $(n=8)$. D, E The effect of MOOs on the immobility time (D) and the climbing time $(E)$ in the FST test $(n=8)$. F The effect of MOOs on the sucrose preference in the SPT test $(n=8)$. G, H The traveling distance $(G)$ and average speed $(H)$ among these 4 groups in the OFT test $(n=8)$. I The weight of 4 groups at the end of the experiment $(n=8)$. ${ }^{*}<0.05,{ }^{*} P<0.01$. PSD, post-stroke depression; tMCAO, transient middle cerebral artery occlusion; CUMS, chronic unpredictable mild stress; Ctrl + v, control group treated with vehicle; Ctrl + M, control group treated with MOOs; PSD + v, PSD group treated with vehicle; PSD + M, PSD group treated with MOOs; TST, tail suspension test; FST, forced swimming test; SPT, sucrose preference test; OFT, open field test. 
A

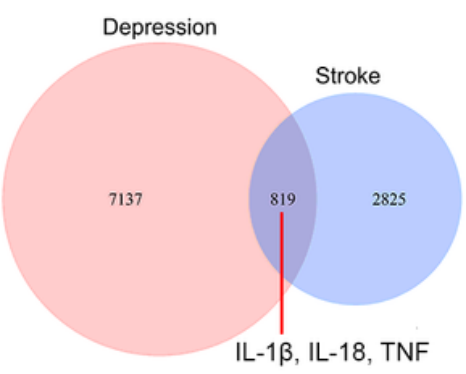

C

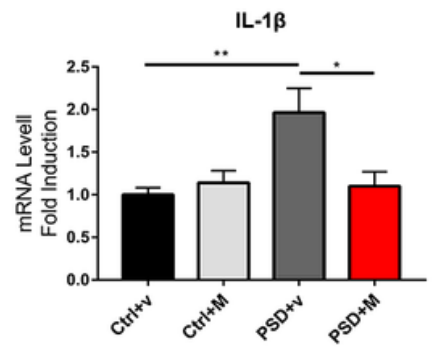

B

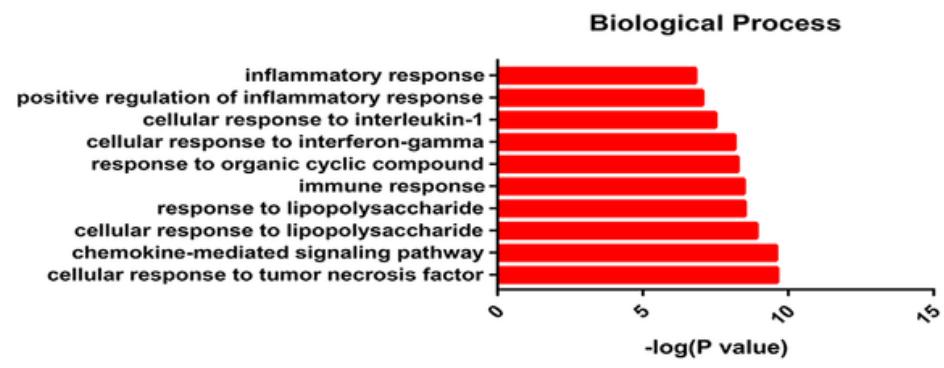

E

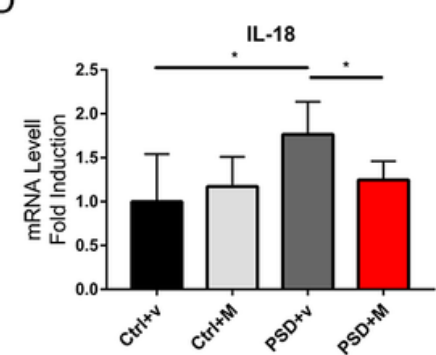

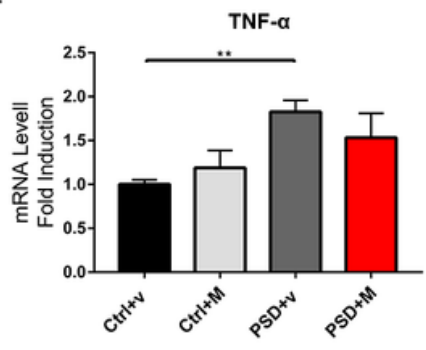

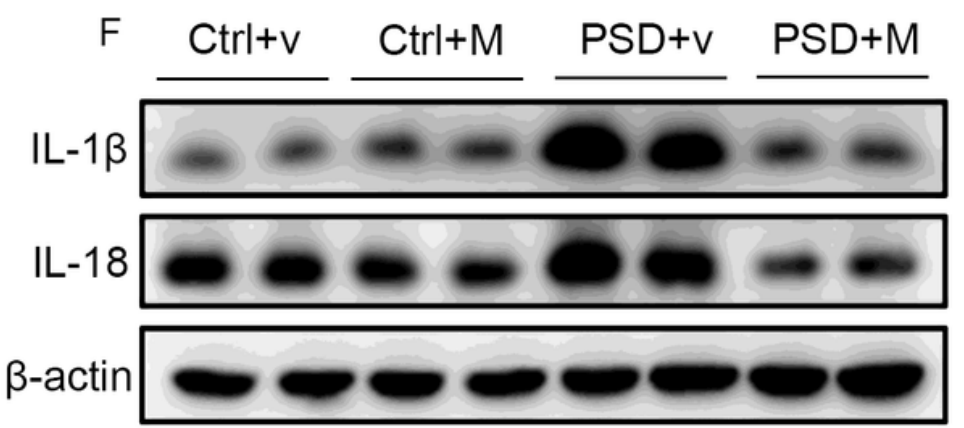

G

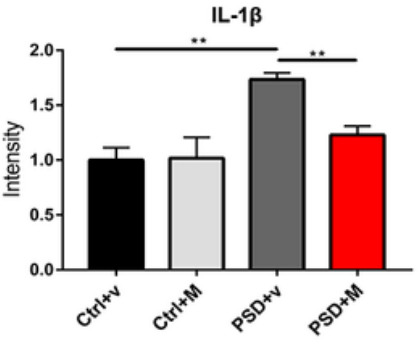

$\mathrm{H}$

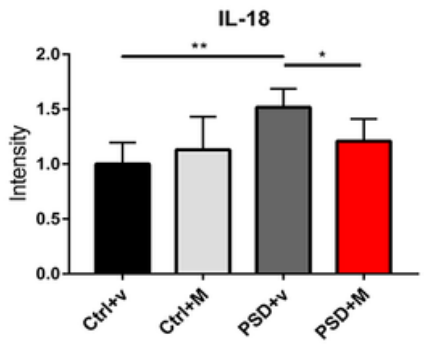

\section{Figure 2}

Morinda officinalis oligosaccharides (MOOs) inhibit the inflammatory response in the hippocampus of PSD rats. A Venn diagram comparing two lists of DEGs in the brain of rats with stroke or depression. $B$ The top biological process GO terms of the overlapping DEGs. C-E Quantification of the IL-1 1 (C), IL-18 (D) and TNF-a (E) mRNA expression in the hippocampus of rats treated with vehicle or MOOs by RT-PCR $(n=6)$. F-H Western blot analysis showing IL-1 $(G)$ and IL-18 $(H)$ expression in the hippocampus of rats treated with vehicle or MOOs $(n=6) .{ }^{*}<0.05, * * P<0.01$. PSD, poststroke depression; DEGs, differentially expressed genes; Ctrl + v, control group treated with vehicle; $\mathrm{Ctrl}+\mathrm{M}$, control group treated with MOOs; PSD + v, PSD group treated with vehicle; PSD + M, PSD group treated with MOOs. 
A

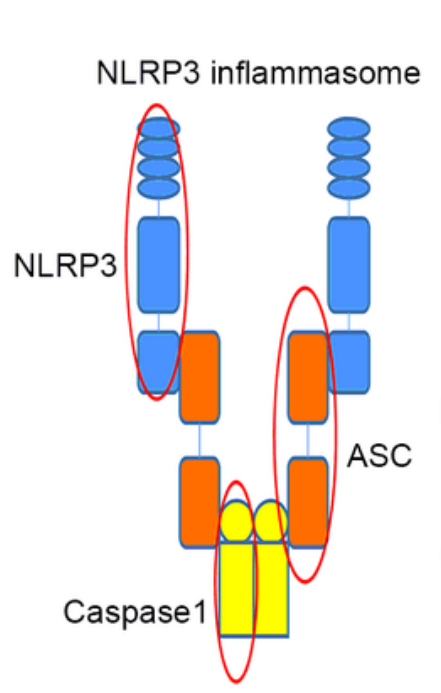

G

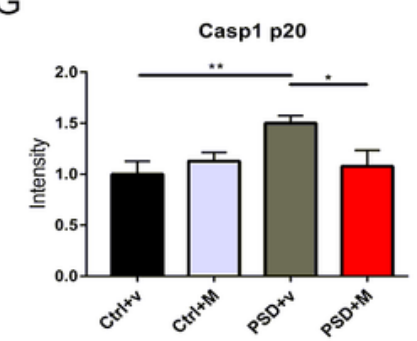

$\mathrm{H}$

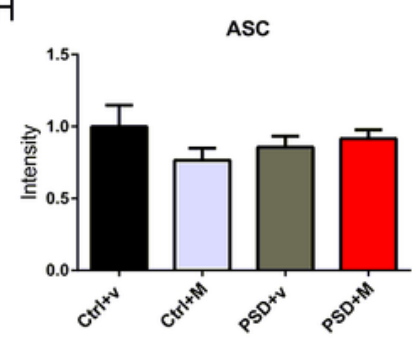

B

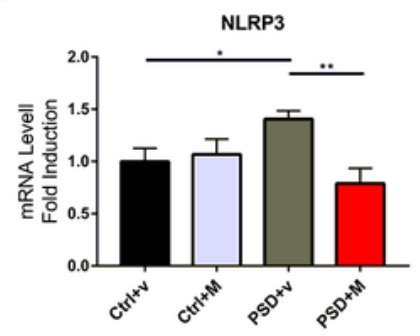

C

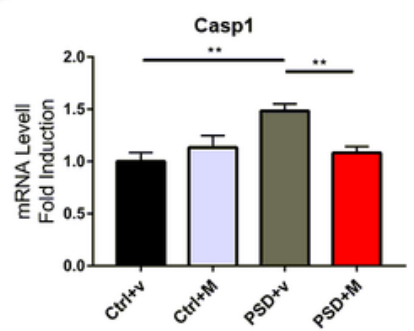

D

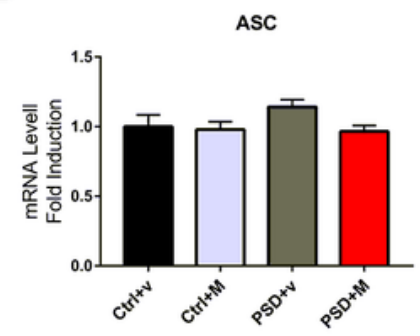

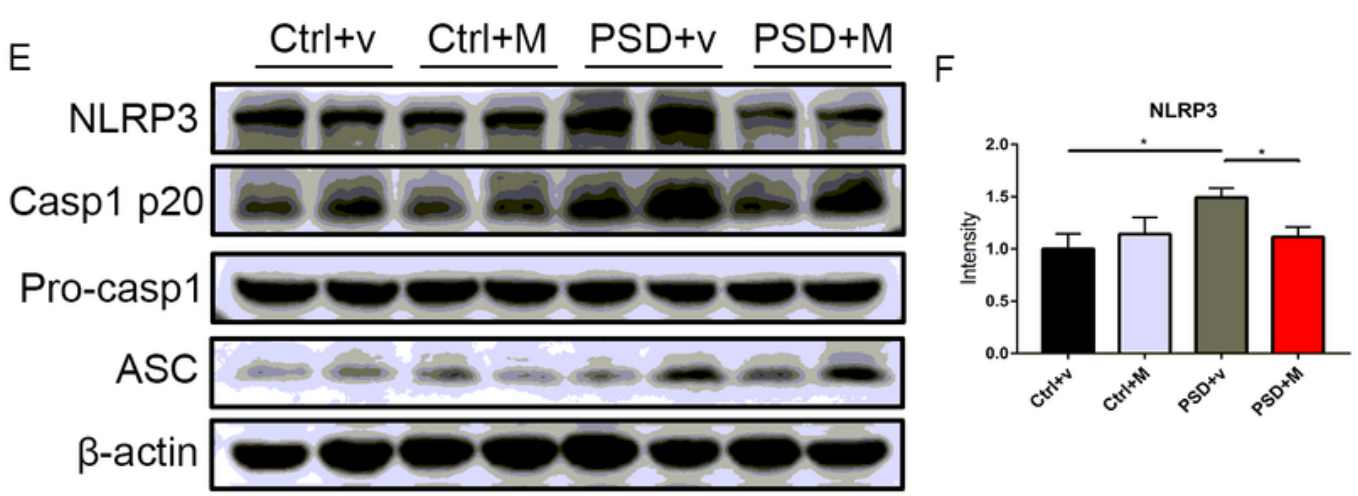

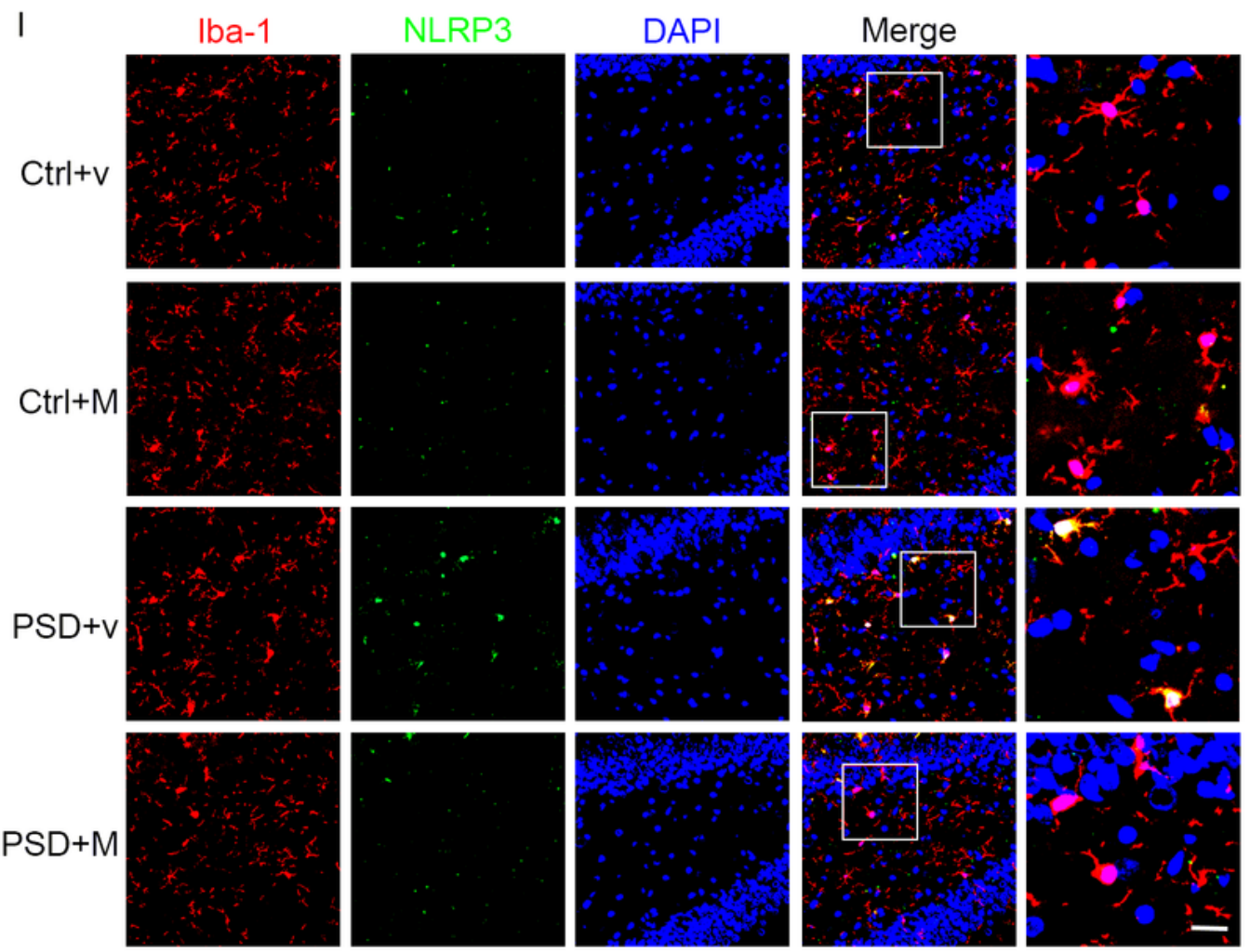

Figure 3

Morinda officinalis oligosaccharides (MOOs) suppress the microglial NLRP3 inflammasome activation in the hippocampus of PSD rats. A A presentative diagram of NLRP3 inflammasome. B-D Quantification of the NLRP3 (B), Caspase1 (C) and ASC (D) mRNA expression in the hippocampus of rats treated with vehicle or MOOs by RT-PCR ( $n=6)$. E-H Western blot analysis showing NLRP3 (F), Caspase1 (G) and ASC (H) expression in hippocampus of rats treated with vehicle or MOOs $(n=6)$. I Immunofluorescence 
staining showing the expression of NLRP3 in the hippocampus (green). Microglia were stained with Iba-1 (red). Scale bar, $15 \mu \mathrm{m}$. ${ }^{*} P<0.05,{ }^{\star} \mathrm{P}<0.01$. NLRP3, nucleotide-binding domain leucine-rich repeat and pyrin domain containing receptor 3; Casp1, Caspase1; ASC, apoptosis-associated speck-like protein containing a caspase recruitment domain; PSD, post-stroke depression; Ctrl + v, control group treated with vehicle; $\mathrm{Ctrl}+\mathrm{M}$, control group treated with MOOs; PSD + v, PSD group treated with vehicle; PSD + M, PSD group treated with MOOs; Iba-1, ionised calcium binding adaptor molecule 1.

A

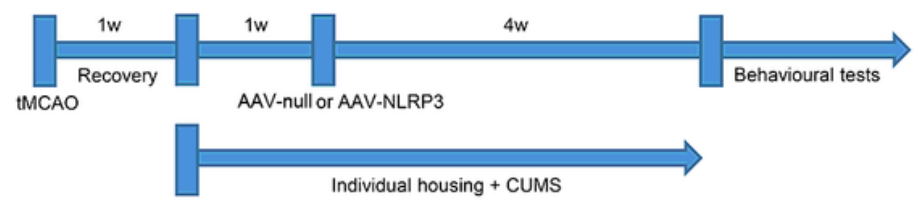

D

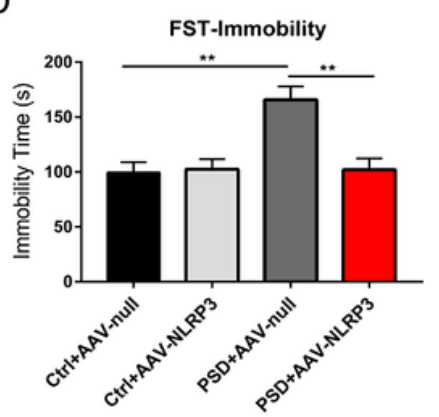

$\mathrm{H}$

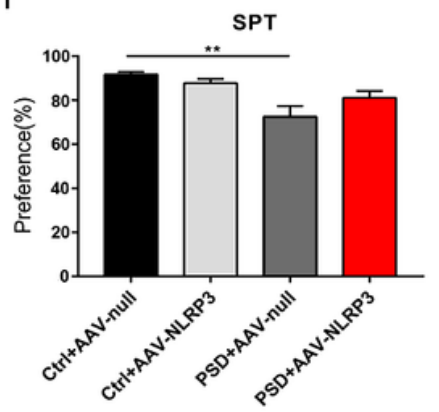

$E$

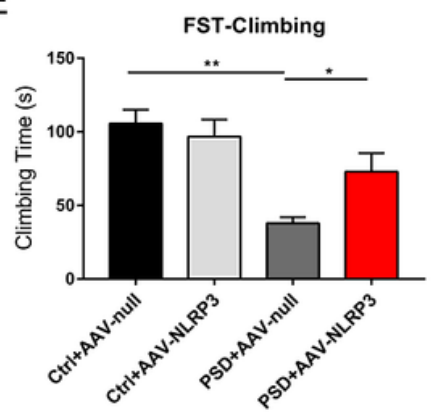

I

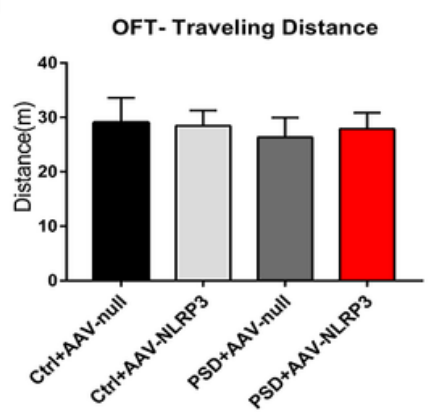

B

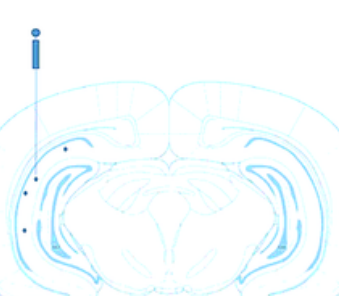

$\mathrm{F}$

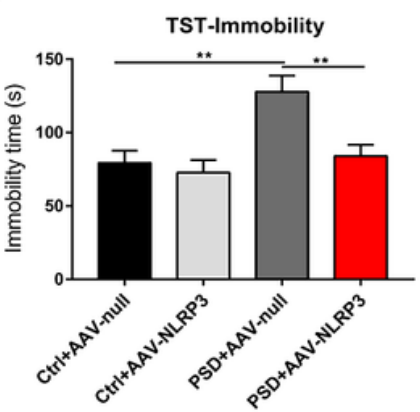

$\mathrm{J}$

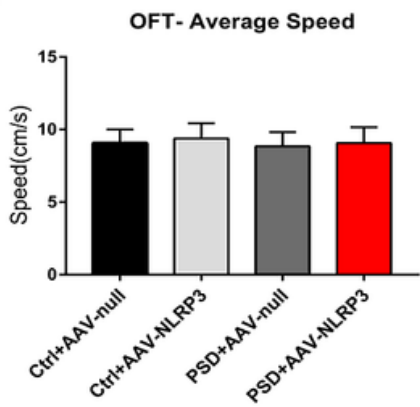

C

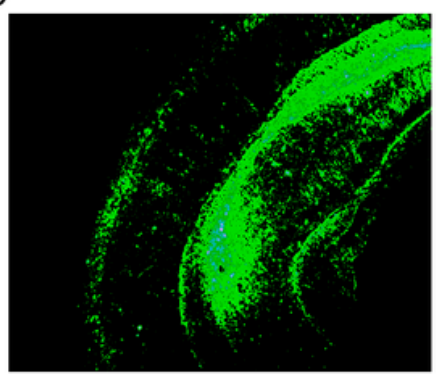

G

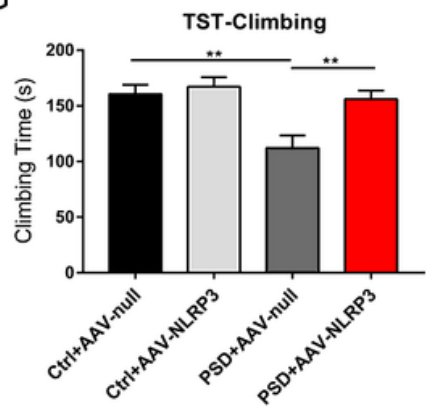

$\mathrm{K}$

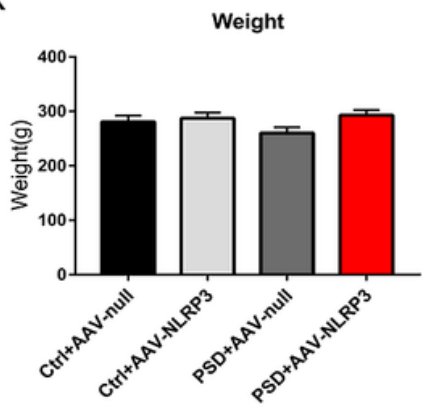

Figure 4

NLRP3 downregulation ameliorates depressive-like behaviors in PSD rats. A An illustration of the experimental schedule. B Illustration of the adeno-associated virus (AAV) injection in rat brain. C Representative image shows the AAV-infected hippocampal area in rat brain. D, E The effect of AAVinduced NLRP3 downregulation on the immobility time (D) and the climbing time (E) in the TST test $(n=8)$. F, G The effect of AAV-induced NLRP3 downregulation on the immobility time $(F)$ and the climbing time $(G)$ in the FST test $(n=8)$. H The effect of AAV-induced NLRP3 downregulation on the sucrose preference in the SPT test $(n=8)$. I, J The traveling distance $(I)$ and average speed $(J)$ among these 4 groups in the OFT test $(n=8)$. K The weight of 4 groups at the end of the experiment $(n=8)$. *P<0.05, **P< 
0.01. PSD, post-stroke depression; $\mathrm{TMCAO}$, transient middle cerebral artery occlusion; CUMS, chronic unpredictable mild stress; Ctrl + AAV-null, control group with AAV-null injection; Ctrl + AAV-NLRP3, control group with AAV-NLRP3 injection; PSD + AAV-null, PSD group with AAV-null injection; PSD + AAV-NLRP3, PSD group with AAV-NLRP3 injection; TST, tail suspension test; FST, forced swimming test; SPT, sucrose preference test; OFT, open field test.
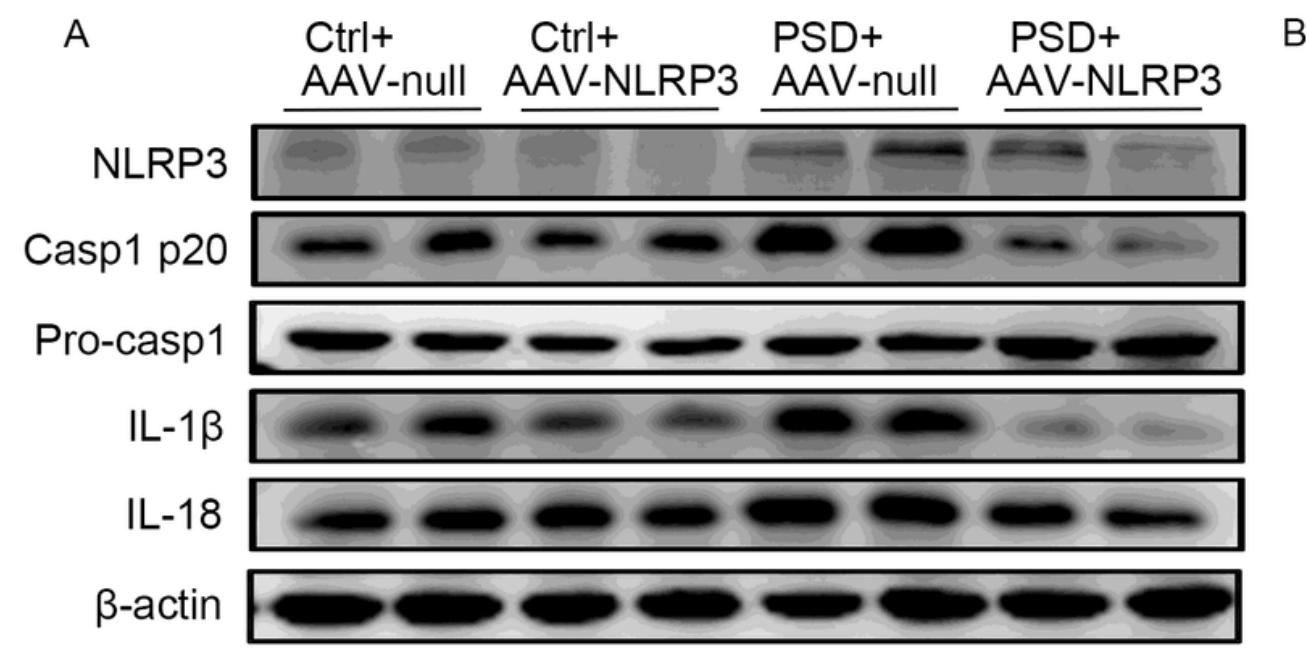

$B$

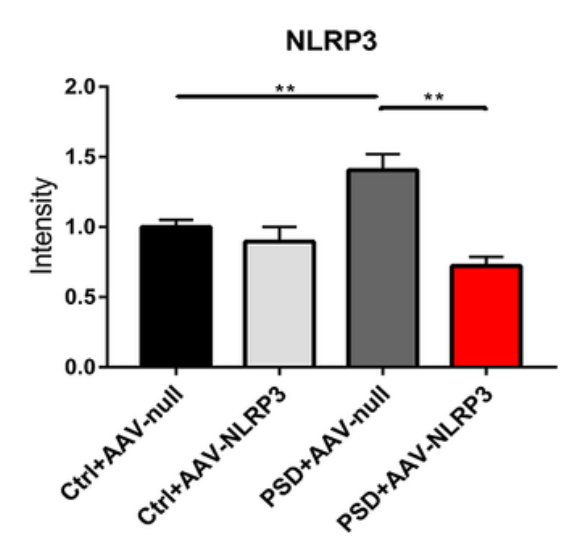

C

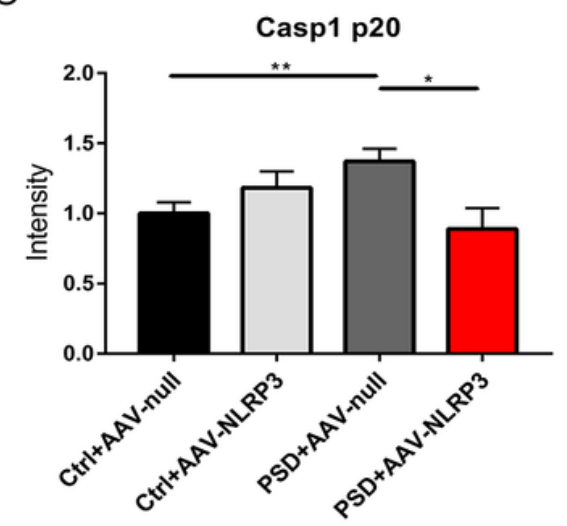

D

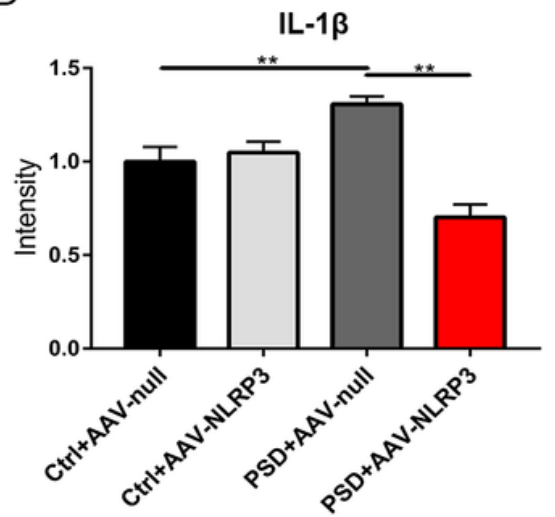

$\mathrm{E}$

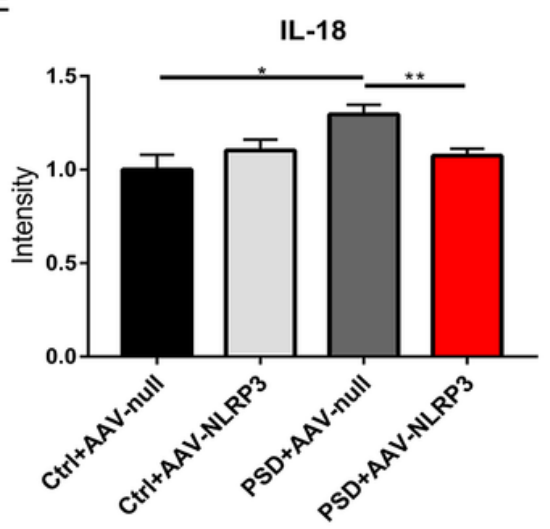

\section{Figure 5}

NLRP3 downregulation alleviates hippocampal inflammation response in PSD rats. A-E Western blot analysis of the expression of NLRP3 (B), Caspase1 (C), IL-1ß (D) and IL-18 (E) in the hippocampus of rats infected with AAV-null or AAV-NLRP3 $(n=6)$. $P<0.05$, $* * P<0.01$. PSD: post-stroke depression; Ctrl + AAV-null, control group with AAV-null injection; Ctrl + AAV-NLRP3, control group with AAV-NLRP3 injection; PSD + AAV-null, PSD group with AAV-null injection; PSD + AAV-NLRP3, PSD group with AAV-NLRP3 injection. 
$\begin{array}{cccccc}\operatorname{MOOs}(\mathrm{mg} / \mathrm{ml}) & - & - & 1.25 & 2.5 & 5 \\ \text { LPS }+A T P & - & + & + & + & +\end{array}$

A
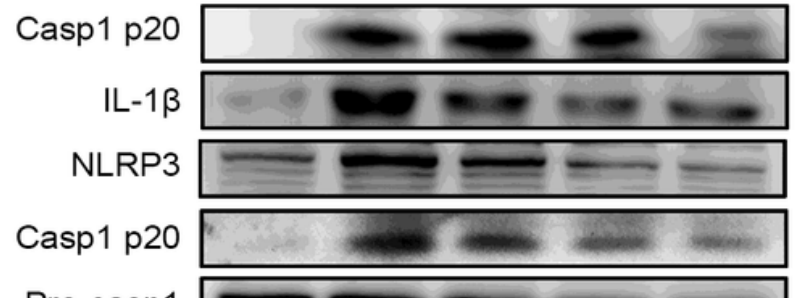

Pro-casp1

IL-1 $1 \beta$

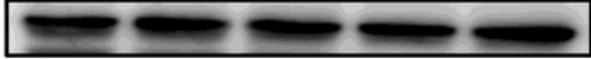

IL-18

$\beta$-actin

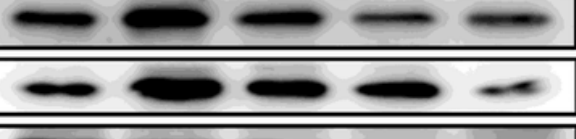

Lys

B

SN

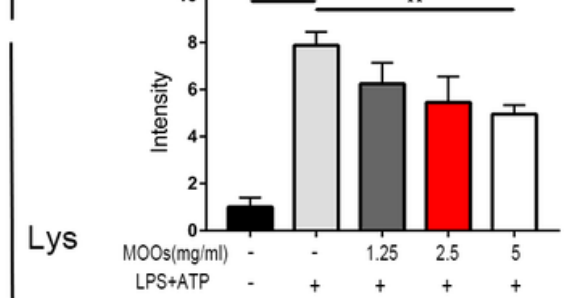

D

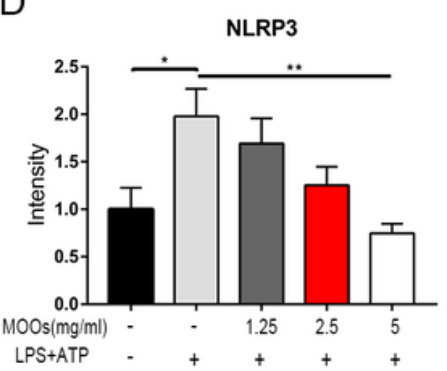

E

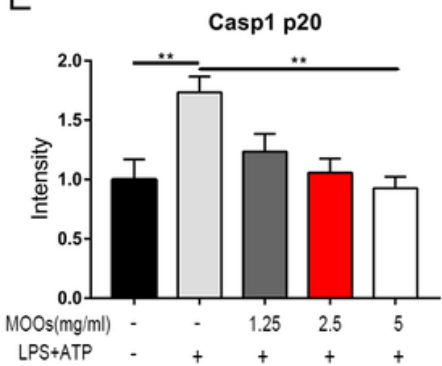

$\mathrm{F}$

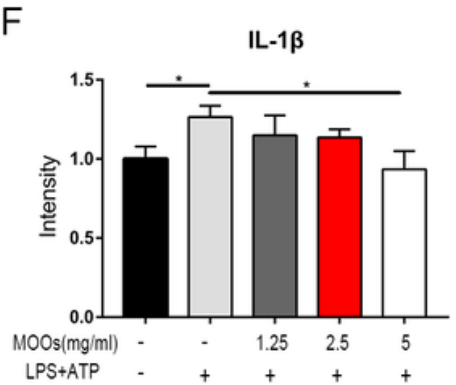

C

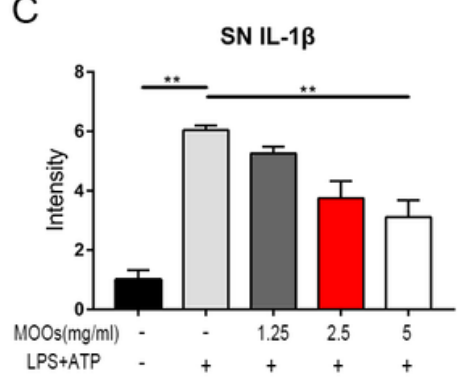

G

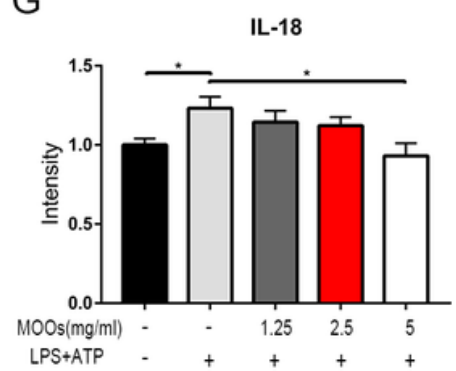

Figure 6

Morinda officinalis oligosaccharides (MOOs) inhibit LPS+ATP-induced NLRP3 inflammasome activation in BV2 cells. A-G Western blot analysis of the expression of Caspase1 (B), IL-1 $\beta$ (C) in supernatants and NLRP3 (D), Caspase1 (E), IL-1 $\beta$ (F) and IL-18 (G) in BV2 cell Lys after ATP+LPS and/or MOOs treatment (n $=6) .{ }^{\star} P<0.05,{ }^{\star} * \mathrm{P}<0.01$. SN, supernatants. 
A
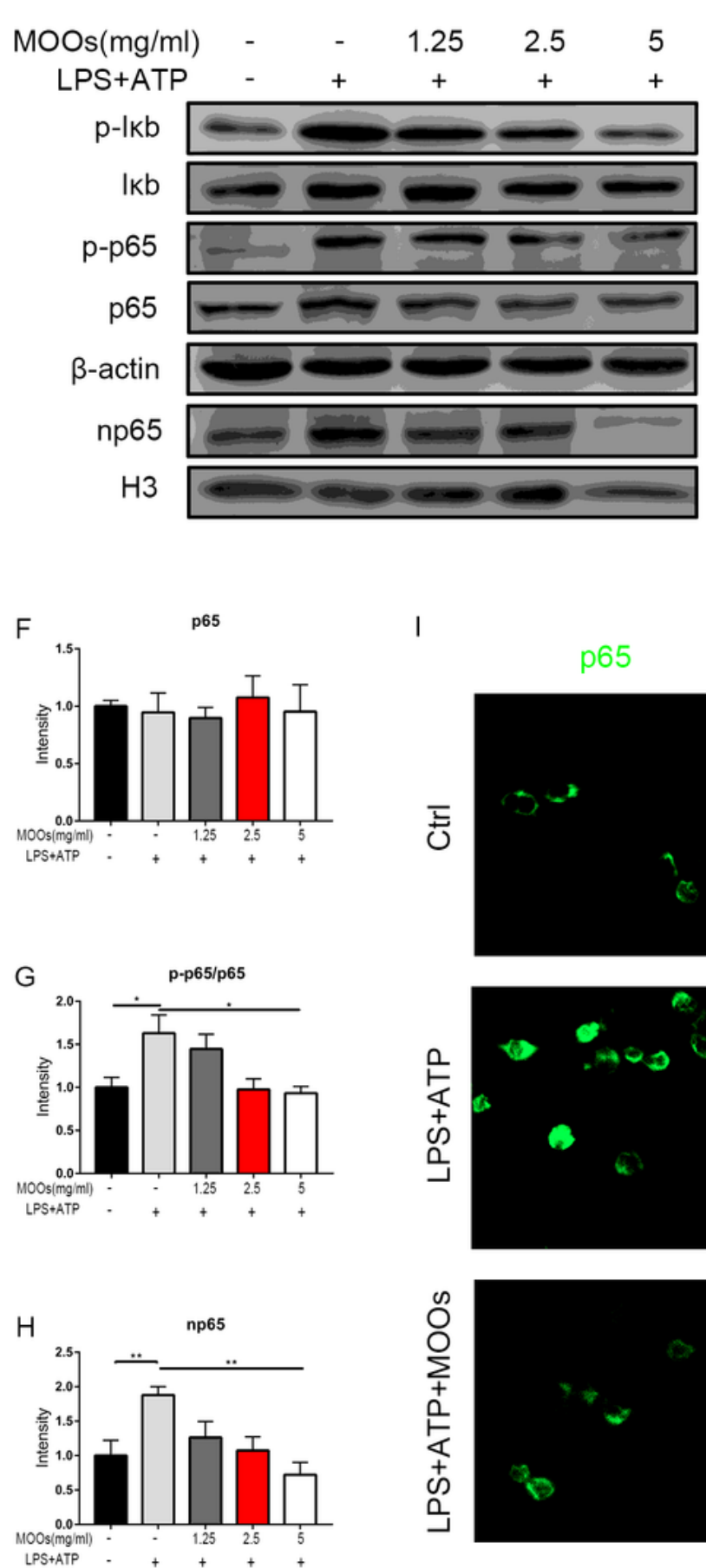

B
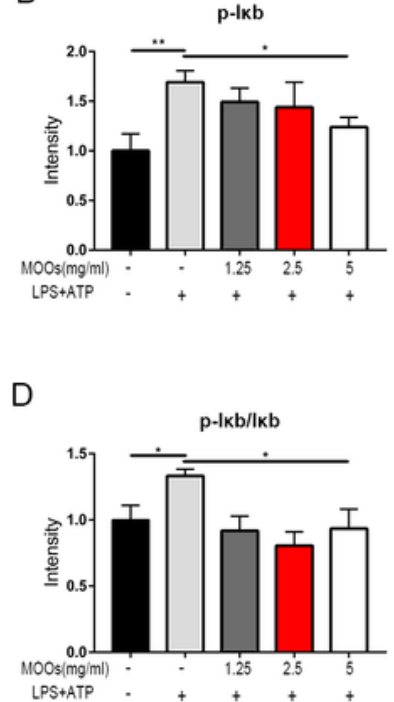

E
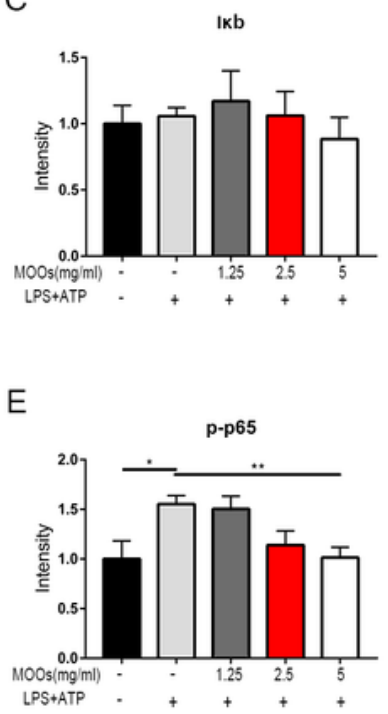

Merge
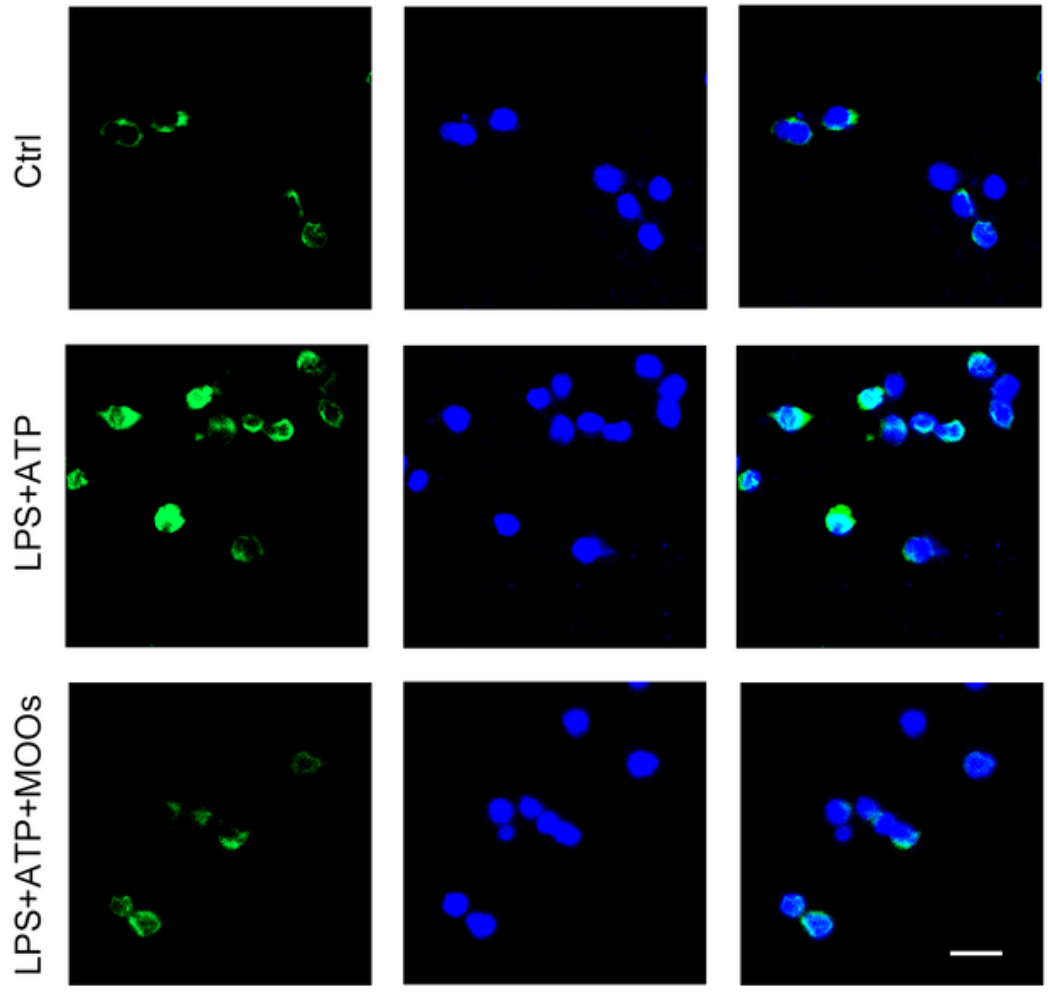

\section{Figure 7}

Morinda officinalis oligosaccharides (MOOs) suppress NF-KB signaling pathway in LPS+ATP treated BV2 cells. A-H Western blot analysis of the expression of p-ІкB (B), IKB (C), p-p65 (E), p65 (F) and nuclear p65 $(\mathrm{np65})(\mathrm{H})$ in BV2 cell after ATP+LPS and/or MOOs treatment $(\mathrm{n}=6)$. I Representative immunofluorescence images showing the translocation of p65 into nucleus. In the LPS+ATP+MOOs 
group, BV2 cells were treated with MOOs at the concentration of $5 \mathrm{mg} / \mathrm{mL}$. Scale bar, $20 \mu \mathrm{m}$. ${ }^{*} \mathrm{P}<0.05$, $\star * P<0.01$.
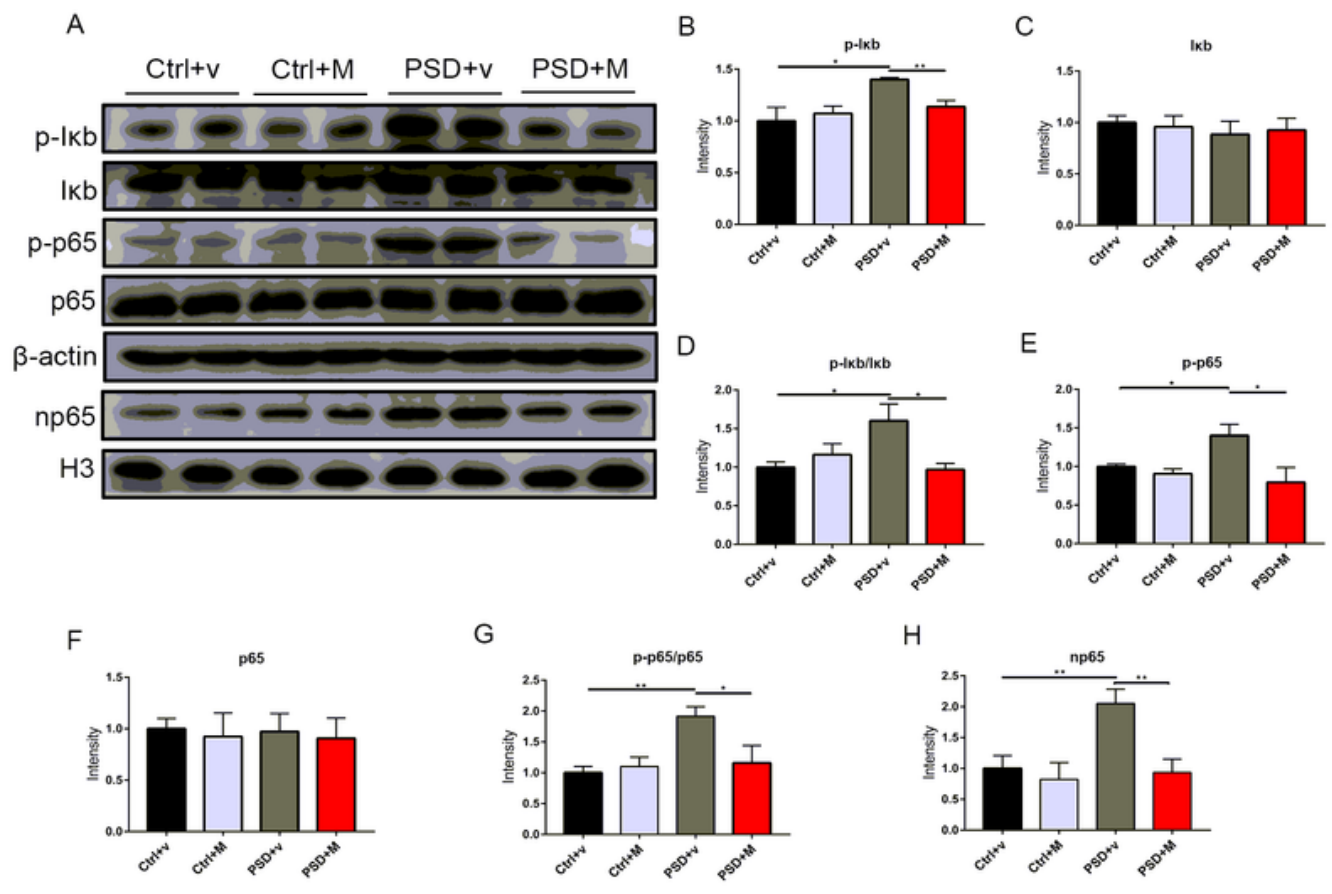

$\mathrm{H}$
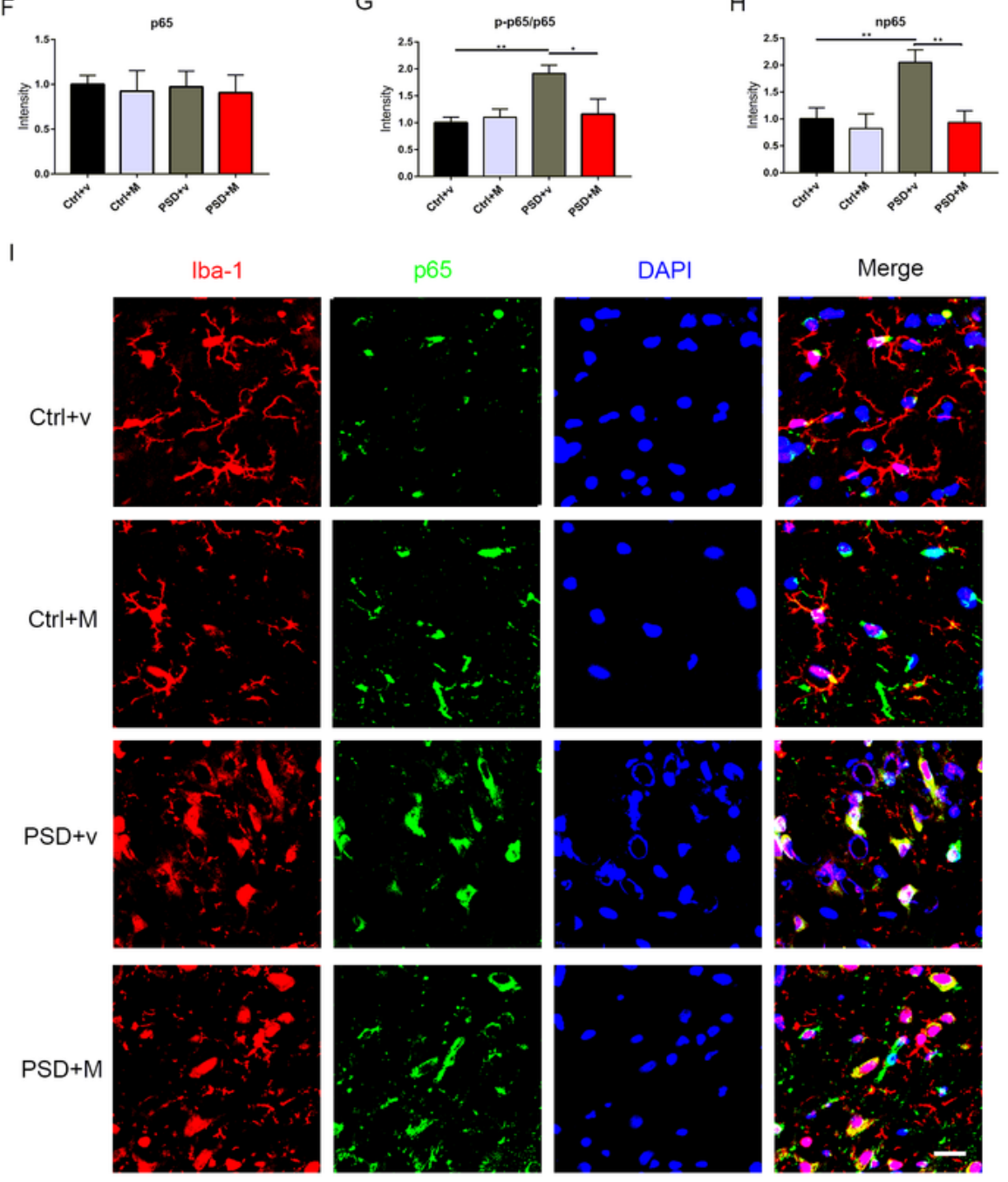

Figure 8

Morinda officinalis oligosaccharides (MOOs) suppress NF-KB signaling pathway in PSD rats. A-H Western blot analysis of the expression of p-ІкB (B), IKB (C), p-p65 (E), p65 (F) and nuclear p65 (np65) (H) in the 
hippocampus of rats treated with vehicle or MOOs $(n=6)$. I Representative immunofluorescence images showing the translocation of p65 into nucleus in microglia. Scale bar, $15 \mu \mathrm{m} .{ }^{*} \mathrm{P}<0.05,{ }^{\star \star} \mathrm{P}<0.01$. 